\title{
A kinetic Fokker-Planck approach to model hard-sphere gas mixtures
}

Cite as: Phys. Fluids 32, 027103 (2020); https://doi.org/10.1063/1.5141909

Submitted: 09 December 2019 . Accepted: 30 January 2020 . Published Online: 18 February 2020

C. Hepp, (D. Grabe, and K. Hannemann
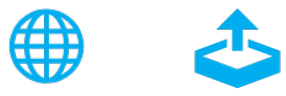

\section{ARTICLES YOU MAY BE INTERESTED IN}

A kinetic Fokker-Planck approach for modeling variable hard-sphere gas mixtures

AIP Advances 10, 085219 (2020); https://doi.org/10.1063/5.0017289

Direct simulation Monte Carlo on petaflop supercomputers and beyond

Physics of Fluids 31, 086101 (2019); https://doi.org/10.1063/1.5108534

Non-equilibrium parameter for a hybrid Fokker-Planck/DSMC scheme

AIP Conference Proceedings 2132, 070002 (2019); https://doi.org/10.1063/1.5119556

\section{Physics of Fluids GALLERY OF GOVERS}

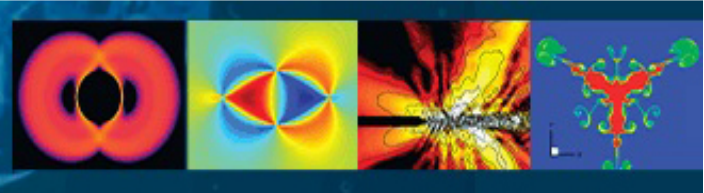




\title{
A kinetic Fokker-Planck approach to model hard-sphere gas mixtures
}

\author{
Cite as: Phys. Fluids 32, 027103 (2020); doi: 10.1063/1.5141909 \\ Submitted: 9 December 2019 - Accepted: 30 January 2020 • \\ Published Online: 18 February 2020
}

\section{Hepp, ${ }^{\text {a) }}$ (D) Mrabe, (D) and K. Hannemann}

\author{
AFFILIATIONS \\ German Aerospace Center (DLR), Bunsenstraße 10, D-37073 Göttingen, Germany
}

a) Author to whom correspondence should be addressed: Christian.Hepp@dlr.de

\begin{abstract}
Since its first introduction, it has always been a subject of research to find models for a meaningful approximation of the highly accurate but complex Boltzmann equation. In the kinetic Fokker-Planck (FP) approach, a FP operator in velocity space is employed to approximate the collision integral of the Boltzmann equation. Instead of directly solving the resulting FP equation, a Monte Carlo technique is used to model an associated random process. This approach leads to an efficient stochastic solution algorithm. In recent years, the FP ansatz has become increasingly popular. Nevertheless, the modeling of gas mixtures in the context of kinetic FP has so far only been addressed in a very few papers. This article introduces a kinetic FP model that is capable of describing gas mixtures with particles interacting according to the hard-sphere collision model. The model is constructed to reproduce Grad's 13 moment equations on a Navier-Stokes level of accuracy for gas mixtures with an arbitrary number of constituents. A stochastic simulation algorithm is derived that ensures a correct evolution of the species diffusion velocities and the species temperatures for a homogeneous gas, regardless of the applied time step size. It is shown that the proposed model is capable of correctly predicting shear stresses, heat fluxes, and diffusion velocities for different test cases, employing a He-Ar mixture.
\end{abstract}

Published under license by AIP Publishing. https://doi.org/10.1063/1.5141909

\section{INTRODUCTION}

Many applications of aerodynamics, for example, the modeling of gas flows in vacuum systems ${ }^{1}$ or reentry flows, ${ }^{2}$ require the modeling of a wide range of rarefaction and nonequilibrium effects. The magnitude of non-equilibrium is often characterized by the Knudsen number $\mathrm{Kn}=\lambda / l$, where $\lambda$ denotes the particle mean free path and $l$ is a characteristic length scale of the regarded problem. When the local Knudsen number is small, many particle collisions occur, which leads to the assumption that the distribution of the thermal particle velocities is near to a local Maxwellian. In this case, the gas flow can be modeled macroscopically by the well-known system of Navier-Stokes equations. On the other hand, a large local Knudsen number may lead to a non-equilibrium velocity distribution function. In such a case, the Navier-Stokes equations lose validity.

Gases in nature may be composed of several particle species. Hence, the modeling of gas mixtures is an essential task for the treatment of a number of aerodynamic problems such as reentry flows, ${ }^{2}$ expansion flows, ${ }^{3}$ or flows in vacuum facilities. ${ }^{1}$ In continuum theory, the treatment of mixtures is usually simplified by several assumptions, such as the postulation of an equalized temperature for all particle species. ${ }^{4}$ However, in strong non-equilibrium flows, these simplifications lose their validity.

In kinetic theory, a gas mixture can be described by a set of Boltzmann equations,

$$
\begin{aligned}
\frac{\partial f^{(\alpha)}}{\partial t} & +v_{k} \frac{\partial f^{(\alpha)}}{\partial x_{k}}+\frac{F_{k}}{m_{\alpha}} \frac{\partial f^{(\alpha)}}{\partial v_{k}} \\
= & \underbrace{2 \pi \sum_{\beta} \iint\left(f^{(\alpha) \prime} f^{(\beta) \prime}-f^{(\alpha)} f^{(\beta)}\right) g^{(\alpha \beta)} b \mathrm{~d} b \mathrm{~d} \mathbf{v}^{(\beta)}}_{S_{\text {Boltz }}^{\alpha}} .
\end{aligned}
$$

The indices $\alpha$ and $\beta$ refer to the different particle species, $b$ denotes the impact parameter for a collision between $\alpha$ and $\beta$ particles, $m^{(\alpha)}$ means the particle mass, $g^{(\alpha \beta)}=\left|\mathbf{v}^{(\beta)}-\mathbf{v}^{(\alpha)}\right|$ is the relative particle velocity, $f^{(\alpha)}\left(\mathbf{x}, \mathbf{v}^{(\alpha)}, t\right)$ is the species particle distribution function before the collision, and $f^{(\alpha) \prime}\left(\mathbf{x}, \mathbf{v}^{(\alpha)}, t\right)$ denotes the species particle distribution function after a collision. Theoretically, Eq. (1) can be 
used to accurately model gas mixtures at arbitrary Knudsen numbers, but due to the high dimensionality of $f^{(\alpha)}\left(\mathbf{x}, \mathbf{v}^{(\alpha)}, t\right)$ and the complexity of the collision integral, the direct solution becomes a computationally expensive task.

An alternative way to model non-equilibrium gas flows features the Direct Simulation Monte Carlo (DSMC) algorithm. ${ }^{6}$ In the DSMC method, the molecular particle motion is calculated directly by a stochastic simulation approach. DSMC is proven to be consistent with the solution of the Boltzmann equation for the monatomic case $^{7}$ and has been validated for the diatomic case. ${ }^{8,9}$ Hence, in the last few decades, the algorithm has become a standard tool for modeling rarefied gas flows. An important requirement of accurate DSMC simulations is the proper resolution of molecular scales: The time step size shall be smaller than the local mean collision time, and the grid cell size shall be smaller than the mean free path. ${ }^{10}$ As a result, the computational effort for DSMC increases strongly as the Knudsen number decreases. This might become a challenge when modeling multiscale gas flows feature a wide range of different local Knudsen numbers.

To resolve this issue, a common practice is to couple DSMC with less accurate but more efficient flow solvers in regions where the resolution of DSMC is not required. One approach to such a hybridization is the coupling between the DSMC algorithm and Navier-Stokes solvers. ${ }^{11,12}$ However, combining DSMC and NavierStokes solvers becomes a challenging task because of the fluctuating boundary conditions for the Navier-Stokes solver, caused by the stochastic nature of the DSMC algorithm. Another approach features the coupling of DSMC with macroscopic particle methods, ${ }^{13-16}$ which calculates the motion of individual gas particles, but without modeling intermolecular collisions. A recent example for such a particle method is the kinetic Fokker-Planck model. ${ }^{1}$

The fundamental approach of the kinetic Fokker-Planck model is the approximation of the Boltzmann equation by a Fokker-Planck equation in velocity space. Instead of obtaining the particle distribution function by solving the Fokker-Planck equation directly, the motion of the underlying particles is modeled by an associated random process. This leads to a particle handling similar to the DSMC algorithm, which allows for a simple coupling of both methods. Jenny et al. ${ }^{13}$ first introduced the linear model, which leads to an incorrect Prandtl number for monatomic gases in the continuum limit. To fix this issue, various authors developed extensions to the linear model. ${ }^{17-20}$ A popular extension is the cubic model by Gorji et al., ${ }^{17}$ which has been extended by methods to model gas mixtures, ${ }^{21}$ polyatomic species, ${ }^{22}$ dense gases, ${ }^{23}$ and efficient integration algorithms. ${ }^{24}$ Gorji and Jenny ${ }^{25}$ also suggested a scheme to efficiently couple the cubic Fokker-Planck model with the DSMC algorithm.

To the best of the authors' knowledge, so far only one paper addresses the modeling of gas mixtures within the kinetic FokkerPlanck approach. ${ }^{21}$ In analogy to the modeling of a single species gas, Gorji and Jenny ${ }^{21}$ approximated the set of Boltzmann equations (1) by a set of Fokker-Planck equations in velocity space. Assuming the Maxwell molecule model, a stochastic solution algorithm is derived that is consistent with time evolution of the first few moments of the Boltzmann equations. All derivations are performed for a binary gas mixture, and the model is tested for a simple Couette flow.
In this paper, we expand the ideas of Gorji and Jenny ${ }^{21}$ to construct a Fokker-Planck model for gas mixtures with particles interacting according to the hard-sphere collision model. The model is designed to reproduce Grad's 13 moment equations on a NavierStokes level of accuracy for gas mixtures with an arbitrary number of constituents. A stochastic simulation algorithm is derived that ensures a correct evolution of the species diffusion velocities and the species temperatures for a homogeneous gas, regardless of the applied time step size. It is shown that the proposed model is capable of predicting the correct shear stresses, heat fluxes, and diffusion velocities for different test cases in a He-Ar mixture.

The structure of this paper is as follows: Sec. II gives a short introduction to the kinetic theory of gas mixtures and the mixture Fokker-Planck model of Gorji and Jenny. ${ }^{21}$ In addition, the DSMC algorithm is briefly outlined, since it is used to perform reference calculation for various test cases. In Sec. III, a Fokker-Planck model for hard sphere (HS) gas mixtures is derived. In Sec. IV, different test cases are investigated. Section $V$ presents the conclusion.

\section{METHODS}

\section{A. Kinetic theory of gas mixtures}

In this section, a brief introduction to the kinetic theory of gas mixtures is given. For further information, the reader is referred to Refs. 5 and 26-28.

The microscopic state of each species is described by a particle distribution function $f^{(\alpha)}\left(\mathbf{x}, \mathbf{v}^{(\alpha)}, t\right)$, describing the number of particles of species $\alpha$ that can be found in a small volume $\mathrm{d} \mathbf{x}$ around $\mathbf{x}$, with velocities in a small range $\mathrm{d} \mathbf{v}^{(\alpha)}$ around $\mathbf{v}^{(\alpha)}$ at time $t$. In the following, superscript indices in brackets refer to different particle species. The distribution functions are normalized to the total number of particles

$$
N^{(\alpha)}=\int_{V} \int f^{(\alpha)}\left(\mathbf{x}, \mathbf{v}^{(\alpha)}, t\right) \mathrm{d} \mathbf{v}^{(\alpha)} \mathrm{d} \mathbf{x}
$$

per species in the system. Here, $V$ denotes the volume of the system. Macroscopic quantities can be calculated as moments of the distribution function. In general, a velocity moment with respect to the distribution function $f^{(\alpha)}$ is defined by

$$
\left\langle\mathbf{g} \mid f^{(\alpha)}\right\rangle=\int f^{(\alpha)}\left(\mathbf{x}, \mathbf{v}^{(\alpha)}, t\right) \mathbf{g}\left(\mathbf{v}^{(\alpha)}\right) \mathrm{d} \mathbf{v}^{(\alpha)}
$$

The reader should note that moments (3) feature a spatial and time dependency that is suppressed for the sake of simplicity. As an example, the species flow velocities are given by

$$
\mathbf{u}^{(\alpha)}(\mathbf{x}, t)=\frac{1}{n^{(\alpha)}}\left\langle\mathbf{v}^{(\alpha)} \mid f^{(\alpha)}\right\rangle,
$$

where $n^{(\alpha)}=\left\langle 1, f^{(\alpha)}\right\rangle$ denotes the species number density. Thermal particle velocities are defined in terms of the species velocities,

$$
\mathbf{c}^{(\alpha)}=\mathbf{v}^{(\alpha)}-\mathbf{u}^{(\alpha)} \text {. }
$$

It is worthwhile to mention that some authors ${ }^{28}$ define thermal velocities,

$$
\hat{\mathbf{c}}^{(\alpha)}=\mathbf{v}^{(\alpha)}-\mathbf{u}
$$


relative to the hydrodynamic velocity of the entire mixture as given by expression (10). However, if not explicitly mentioned, this definition is not further used in the work. Similar to the single species case, species specific temperatures

$$
T^{(\alpha)}(\mathbf{x}, t)=\frac{m^{(\alpha)}}{3 k_{\mathrm{B}} n^{(\alpha)}}\left\langle\mathbf{c}^{(\alpha)} \mathbf{c}^{(\alpha)} \mid f^{(\alpha)}\right\rangle
$$

are defined. The species specific pressure tensors are given by

$$
p_{i j}^{(\alpha)}(\mathbf{x}, t)=m^{(\alpha)}\left\langle c_{i}^{(\alpha)} c_{j}^{(\alpha)} \mid f^{(\alpha)}\right\rangle,
$$

and heat fluxes can be calculated as

$$
\mathbf{q}^{(\alpha)}(\mathbf{x}, t)=m^{(\alpha)}\left\langle\mathbf{c}^{(\alpha)} c_{j}^{(\alpha)} c_{j}^{(\alpha)} \mid f^{(\alpha)}\right\rangle
$$

Here, $m^{(\alpha)}$ mean the mass of an $\alpha$ particle. Averages for the whole mixture are given by summation over species specific moments. An important example features the hydrodynamic flow velocity,

$$
\mathbf{u}(\mathbf{x}, t)=\frac{1}{\rho} \sum_{\alpha}\left\langle\mathbf{u}^{(\alpha)} \mid f^{\alpha}\right\rangle \rho^{(\alpha)},
$$

where $\rho^{(\alpha)}=m^{(\alpha)} \cdot n^{(\alpha)}$ denotes the species mass density and $\rho=\sum_{\alpha} \rho^{(\alpha)}$ the mixture mass density. When calculating the mixture temperature

$$
\frac{3}{2} k_{\mathrm{B}} n T(\mathbf{x}, t)=\sum_{\alpha}\left(\frac{3}{2} k_{\mathrm{B}} n^{(\alpha)} T^{(\alpha)}+\frac{1}{2} \rho^{(\alpha)} \mathbf{u}_{d}^{(\alpha)} \mathbf{u}_{d}^{(\alpha)}\right),
$$

additional terms describing the relative motion of species must be taken into account, where diffusion velocities are given by

$$
\mathbf{u}_{d}^{(\alpha)}=\mathbf{u}^{(\alpha)}-\mathbf{u}
$$

The evolution of distribution functions is described by kinetic models, such as the set of Boltzmann equations (1). Of particular interest is the behavior of kinetic models in the continuum limit. This can be characterized by production terms

$$
P_{\Psi}^{(\alpha)}(X) \equiv m^{(\alpha)} \int S_{\Psi}^{(\alpha)} X \mathrm{~d} \mathbf{c}^{(\alpha)},
$$

where $S_{\Psi}^{(\alpha)}$ refers to a collision operator. Obviously, production terms are directly related to a collision operator. Kinetic models that feature the same lower order production terms $P_{\Psi}^{(\alpha)}\left(c_{i}\right), P_{\Psi}^{(\alpha)}\left(c_{i} c_{j}\right)$, and $P_{\Psi}^{(\alpha)}\left(c_{i} \mathbf{c}^{2}\right)$ show the same characteristics in the continuum limit. Hence, production terms are a powerful indicator for analyzing kinetic models in the limit of small Knudsen numbers.

\section{B. DSMC}

This section briefly summarizes the DSMC method that is applied to perform reference simulation for validating the proposed Fokker-Planck (FP) model. For further details on the method, the reader is referred to Ref. 6.

In the DSMC algorithm, the distribution function $f^{(\alpha)}\left(\mathbf{r}, \mathbf{v}^{(\alpha)}, t\right)$ is approximated by a set of computational particles, typically representing a large number of real atoms or molecules. The ratio between the number of real to simulated particles defines the scaling factor $F_{N}$.

Figure 1 schematically shows a procedure for a standard DSMC simulation. The particles are moved through the domain, and the particle velocities are updated by modeling molecular collisions in a separate step.

Particles are generated at simulation boundaries according to macroscopic inflow conditions. Neglecting external forces, the motion of the particles is described by a linear displacement based on the current particle velocities. To model the collision process, the domain is divided into grid cells and collisions are only performed between particles that are in the same cell. Macroscopic quantities are calculated by averaging over the attributes of the particles in a grid cell.

A crucial part of the algorithm is the modeling of particle collisions. DSMC collisions are modeled in a two-step process. Initially, collision partners are selected. Afterwards, a collision between the selected particle pair is carried out. In order to select collision partners, the "no time counter" (NTC) ${ }^{6}$ scheme is applied. This scheme selects randomly a number of

$$
N_{\text {coll }}=\left\lfloor\frac{1}{2} N_{\mathrm{p}}\left(N_{\mathrm{p}}-1\right) F_{\mathrm{N}} \frac{\left(\sigma_{\mathrm{T}} c_{\mathrm{r}}\right)_{\max }}{V_{\mathrm{c}}} \Delta t+\mathbb{R}\right\rfloor
$$

particle pairs. Here, $N_{\mathrm{p}}$ denotes the number of particles in the cell, $V_{c}$ denotes the cell volume, and $\Delta t$ denotes the time step size. The floor function \lfloor\rfloor and the random number $\mathbb{R} \in[0,1]$ are required to reach a statistical exact collision. The value $\left(\sigma_{\mathrm{T}} c_{\mathrm{r}}\right)_{\max }$ denotes the maximum of the collision probability $\sigma_{\mathrm{T}} c_{\mathrm{r}}$. It is species independent, saved separately for each grid cell and continuously updated during a simulation. The total cross section $\sigma_{\mathrm{T}}$ depends on the collision model and is discussed later in this section. $c_{\mathrm{r}}$ refers to the relative particle velocity. Each particle pair is tested for a collision with probability

$$
P_{\text {coll }}=\frac{\sigma_{\mathrm{T}} c_{\mathrm{r}}}{\left(\sigma_{\mathrm{T}} c_{\mathrm{r}}\right)_{\max }}
$$

Once a particle pair has been selected for a collision, particle velocities are adjusted accordingly. For this, certain collision models can be employed. To be consistent with the FP model, this work applies the simple hard sphere (HS) model that describes particles as hard spheres. Hence, the intermolecular force is completely characterized

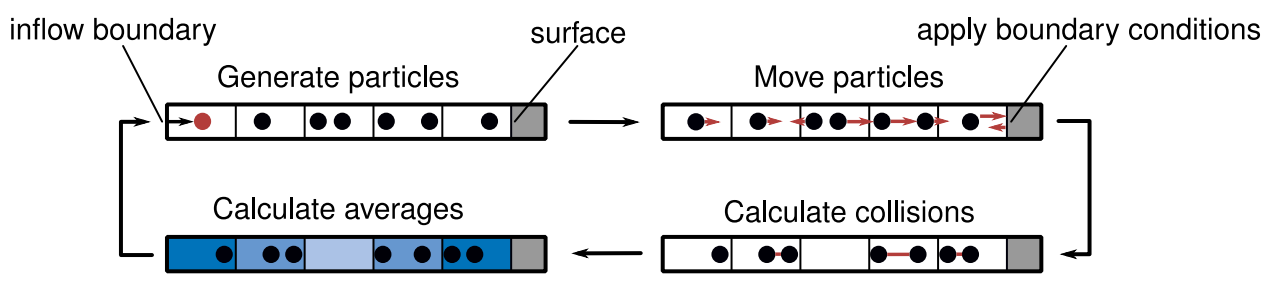

FIG. 1. Typical DSMC simulation loop for a simple 1-D case. 
by a distance $d^{(\alpha-\beta)}=\left(d^{(\alpha)}+d^{(\beta)}\right) / 2$, where $d^{(\alpha)}$ and $d^{(\beta)}$ characterize the diameters of the collision partners. The total collision cross section

$$
\sigma_{T}=\frac{d_{\mathrm{ref}}^{(\alpha-\beta), 2}}{4}
$$

is a constant, and the scattering angle is isotropic in the center of mass reference.

Various other collision models can also be used for describing more complex collision phenomena, for example, the variable hard sphere, ${ }^{29}$ the variable soft sphere, ${ }^{30,31}$ or the generalized hard sphere model. ${ }^{32}$ However, since they are not used in this work, they will not be discussed further.

Due to splitting of the particle motion and collisions, the cell size should be smaller than the smallest species local mean free path and the time step size shall be less than the smallest species mean collision time.

For a single species gas, it has been proven that the distribution function, which is represented by DSMC particles, is consistent with the solution of the Boltzmann equation. ${ }^{7}$ Although there is no such strict proof for multi-species flow, the similarity with the single species case suggests a similar relationship for gas mixtures. In this work, the DSMC algorithm is applied to generate reference solutions for test cases studied in Sec. IV.

\section{Cubic Fokker-Planck}

This section outlines the cubic Fokker-Planck model for a two component gas mixture of Maxwell molecules, as derived by Gorji and Jenny. ${ }^{21}$ The components of the mixture are referred to by indices $\alpha$ and $\beta$. For the sake of simplicity, but without loss of generality, all equations are given only for species $\alpha$. Equations for species $\beta$ can be obtained by simply exchanging the indices $\alpha$ and $\beta$.

Similar to monatomic Fokker-Planck models, the set of Boltzmann equations (1) is approximated by a set of Fokker-Planck equations

$$
\begin{aligned}
\frac{\partial f^{(\alpha)}}{\partial t} & +v_{i}^{(\alpha)} \frac{\partial f^{(\alpha)}}{\partial x_{i}}+\frac{F_{i}^{(\alpha)}}{m^{(\alpha)}} \frac{\partial f^{(\alpha)}}{\partial v_{i}^{(\alpha)}} \\
= & \underbrace{-\frac{\partial\left(f^{(\alpha)} A_{i \mid \mathrm{MAX}}^{(\alpha)}\right)}{\partial v_{i}^{(\alpha)}}+\frac{\partial^{2}\left(f^{(\alpha)} D_{\mid \mathrm{MAX}}^{(\alpha)}\right)}{\partial v_{i}^{(\alpha)} \partial v_{i}^{(\alpha)}}}_{S_{\mathrm{FP} \mid \mathrm{MAX}}^{(\alpha)}}
\end{aligned}
$$

in velocity space. Here, $S_{\mathrm{FP} \mid \mathrm{MAX}}^{(\alpha)}$ designates the Fokker-Planck operator and $F_{i}^{(\alpha)}$ denotes an external force. The drift coefficient $A_{i \mid \mathrm{MAX}}^{(\alpha)}$ and diffusion coefficient $D_{\mid \mathrm{MAX}}^{(\alpha)}$ are chosen separately for each species. A cubic ansatz

$$
\begin{aligned}
A_{i \mid \mathrm{MAX}}^{(\alpha)} \equiv & b_{i}^{(\alpha)}+\psi_{i j}^{(\alpha)} c_{j}^{(\alpha)}+\gamma_{i}^{(\alpha)}\left(c_{j}^{(\alpha)} c_{j}^{(\alpha)}-\left\langle c_{j}^{(\alpha)} c_{j}^{(\alpha)} \mid f^{(\alpha)}\right\rangle\right) \\
& +\Lambda^{(\alpha)}\left(c_{i}^{(\alpha)} c_{j}^{(\alpha)} c_{j}^{(\alpha)}-\left\langle c_{i}^{(\alpha)} c_{j}^{(\alpha)} c_{j}^{(\alpha)} \mid f^{(\alpha)}\right\rangle\right)
\end{aligned}
$$

in terms of thermal particle velocities is used for the drift coefficient. The constant $\Lambda^{(\alpha)}$ is chosen to ensure the stability of the model. ${ }^{17}$ The model parameters $b_{i}^{(\alpha)}, \psi_{i j}^{(\alpha)}, \gamma_{i}^{(\alpha)}$, and the diffusion coefficient $D_{\mid \text {MAX }}^{(\alpha)}$ are chosen so that the Fokker-Planck operator $S_{\mathrm{FP} \mid \mathrm{MAX}}^{(\alpha)}$ reproduces lower order Boltzmann production terms,

$$
\begin{gathered}
P_{\mathrm{FP} \mid \mathrm{MAX}}^{(\alpha)}(X) \stackrel{!}{=} P_{\mathrm{Boltz}}^{(\alpha)}(X), \\
X \in\left\{c_{i}^{(\alpha)}, c_{i}^{(\alpha)} c_{i}^{(\alpha)}, c_{<i}^{(\alpha)} c_{j>}^{(\alpha)}, c_{i}^{(\alpha)} c_{j}^{(\alpha)} c_{j}^{(\alpha)}\right\} .
\end{gathered}
$$

The brackets $\langle\ldots\rangle$ refer to the deviatoric part of the tensor $c_{i}^{(\alpha)} c_{j}^{(\alpha)}$. It is worth noting that the right side of expression (19) does not vanish for $X=c_{i}^{(\alpha)}$ and $X=c_{i}^{(\alpha)} c_{i}^{(\alpha)}$ since energy and momentum can be transferred between species. The production terms on the right hand side of (19) are evaluated for binary Maxwell mixtures in Ref. 33. The parameters $b_{i}^{(\alpha)}$ and $D_{\mid \mathrm{MAX}}^{(\alpha)}$ are set so that the linear part of expression (18) satisfies (19) for $X \in\left\{c_{i}^{(\alpha)}, c_{i}^{(\alpha)} c_{i}^{(\alpha)}\right\}$. The nine remaining model parameters $\psi_{i j}^{(\alpha)}, \gamma_{i}^{(\alpha)}$ are determined as a solution of a system of nine linear equations that can be derived out of expression (19) for $X \in\left\{c_{<i}^{(\alpha)} c_{j>}^{(\alpha)}, c_{i}^{(\alpha)} c_{j}^{(\alpha)} c_{j}^{(\alpha)}\right\}$.

Particle trajectories are calculated by integrating a species depended system of the stochastic equation of motions,

$$
\begin{gathered}
\mathrm{d} \mathbf{X}=\mathbf{V}_{i}^{(\alpha)} \mathrm{d} t \\
\mathrm{~d} \mathbf{V}^{(\alpha)}=\mathbf{A}^{(\alpha)} \mathrm{d} t+\sqrt{2 D^{(\alpha)}} \mathrm{d} \mathbf{W}^{(\alpha)}+\mathbf{F}^{(\alpha)} .
\end{gathered}
$$

Here, $\mathbf{X}$ and $\mathbf{V}$ denote particle position and velocity, respectively, and $\mathrm{dW}^{(\alpha)}$ denotes a Wiener process with zero expectation and $\left\langle\mathrm{d} W_{i}^{(\alpha)} \mathrm{d} W_{j}^{(\alpha)}\right\rangle=\delta_{i j}$. In the limit of an infinite number of particles, which are simulated according to (20) and (21), the distribution of the particle positions and velocities corresponds to the distribution function as described by the FP equation (17). For details about the solution of (20) and (21), the reader is referred to Ref. 21.

\section{FOKKER-PLANCK MODEL FOR HARD-SPHERE MIXTURES}

This section describes an approach for modeling gas mixtures within the kinetic Fokker-Planck method. The approach is based on the method of Gorji and Jenny, ${ }^{21}$ who derived a model for describing binary gas mixtures, assuming that particles interact with a mathematical simple but physical unrealistic Maxwell molecular model. However, the model presented in this work features two major improvements compared to the work of Gorji and Jenny. ${ }^{21}$ The proposed model can describe not only binary gas mixtures but also mixtures with an arbitrary number of species. In addition, particle interaction is modeled through the hard-sphere (HS) collision model. Compared to the Maxwell molecule model, the HS model can be advantageous, since it can better catch transport properties for many gases. The HS model is also able to describe the thermodiffusion effect, which is not the case with the Maxwell molecule model. ${ }^{34}$ The influence of thermodiffusion will be discussed in more detail in Sec. IV C. 
Similar to the model described in Sec. II C, the set of Boltzmann equations (1) is approximated by the following set of Fokker-Planck equations:

$$
\frac{\partial f^{(\alpha)}}{\partial t}+v_{i}^{(\alpha)} \frac{\partial f^{(\alpha)}}{\partial x_{i}}+\frac{F_{i}^{(\alpha)}}{m^{(\alpha)}} \frac{\partial f^{(\alpha)}}{\partial v_{i}^{(\alpha)}}=\underbrace{-\frac{\partial\left(f^{(\alpha)} A_{i}^{(\alpha)}\right)}{\partial v_{i}^{(\alpha)}}+\frac{\partial^{2}\left(f^{(\alpha)} D^{(\alpha)}\right)}{\partial v_{i}^{(\alpha)} \partial v_{i}^{(\alpha)}}}_{S_{\mathrm{FP}}^{(\alpha)}} .
$$

The species specific drift coefficients $A_{i}^{(\alpha)}$ and the diffusion coefficients $D^{(\alpha)}$ are constructed so that the Fokker-Planck model gives a correct prediction of the species temperatures, the species mass diffusion, shear stresses, and heat fluxes in the continuum limit. This leads to conservation of mass, momentum, and energy as well as a correct prediction of the transport coefficients for the mixture in the continuum limit. To fulfill these requirements, the FP operator must reproduce the following lower order Boltzmann production terms in the limit of small Knudsen numbers:

$$
\begin{gathered}
P_{\mathrm{Boltz}}^{(\alpha)}(X) \stackrel{!}{=} P_{\mathrm{FP}}^{(\alpha)}(X), \\
X \in\left\{c_{i}^{(\alpha)}, c_{i}^{(\alpha)} c_{i}^{(\alpha)}, c_{<i}^{(\alpha)} c_{j>}^{(\alpha)}, c_{i}^{(\alpha)} c_{j}^{(\alpha)} c_{j}^{(\alpha)}\right\} .
\end{gathered}
$$

The calculation of production terms $P_{\text {Boltz }}^{(\alpha)}(X)$ for the Boltzmann collision operator is, in general, a non-trivial task. Since the FP model is intended to describe particles interacting through the HS collision model, Boltzmann production terms must be derived based on the same assumptions. For the HS collision model, it is, in general, not possible to calculate Boltzmann production terms independently of a distribution function. For example, Gupta ${ }^{28}$ evaluated $P_{\text {Boltz }}^{(\alpha)}(X)$, assuming the HS collision model and a distribution function according to Grad's 13 and 26 moment method. Because Grad's moment method only applies for low Knudsen number gases, the resulting production terms are only valid for near continuum flow. However, since the identity (23) must be true only in the continuum limit, the results of Gupta ${ }^{28}$ may be still applied for this work. In Appendix A, these results are discussed in more detail.

For the FP operator, production terms (13) can be calculated independently of a distribution function. For more details, the reader is referred to Appendix $B$.

Similar to the work of Gorji and Jenny, ${ }^{21}$ a Fokker-Planck operator with a linear drift coefficient is constructed, which correctly predicts the evolution of the species temperatures and the species mass diffusion in the continuum limit. In a second step, the drift coefficient is extended by a higher order expression in the thermal particle velocities, which leads to a correct prediction of the species shear stresses and heat flows in the continuum limit.

\section{A. Linear model}

The drift coefficient $A_{i}^{(\alpha)}$ is chosen so that the model predicts a correct species mass diffusion in the continuum limit. This requirement is satisfied, when expression (23) is fulfilled for $X=c_{i}^{(\alpha)}$. Applying the production terms (A12) and (B10), expression (23) leads to

$$
\begin{aligned}
& -\sum_{\beta=1}^{N} v^{(\alpha \beta)} \mu^{(\beta)}\left[\frac{5}{3} \rho^{(\alpha)}\left(u_{i}^{(\alpha)}-u_{i}^{(\beta)}\right)+\frac{1}{6 \hat{\theta}^{(\alpha \beta)}}\left(\hat{h}_{i}^{(\alpha)}-\frac{\rho^{(\alpha)}}{\rho^{(\beta)}} \hat{h}_{i}^{(\beta)}\right)\right] \\
& \quad \stackrel{!}{=} m^{(\alpha)}\left\langle A_{i}^{(\alpha)} \mid f^{(\alpha)}\right\rangle .
\end{aligned}
$$

The following choice for the drift coefficient $A_{i}^{(\alpha)}$ satisfies (24):

$$
\begin{gathered}
A_{i}^{(\alpha)} \equiv-\sum_{\beta=1}^{N} v^{(\alpha \beta)} \mu^{(\beta)}[\frac{5}{3}\left(\hat{c}_{i}^{(\alpha)}-u_{d, i}^{(\beta)}\right)+\underbrace{\frac{1}{6 \hat{\theta}^{(\alpha \beta)}}\left(\frac{1}{\rho^{(\alpha)}} \hat{h}_{i}^{(\alpha)}-\frac{1}{\rho^{(\beta)}} \hat{h}_{i}^{(\beta)}\right)}_{(*)}] \\
\equiv-s^{(\alpha)} c_{i}^{(\alpha)}+K_{i}^{(\alpha)} .
\end{gathered}
$$

Recall that $\hat{\mathbf{c}}^{(\alpha)}$ denotes the thermal particle velocities as defined by (6). The species heat fluxes $\hat{\mathbf{h}}^{(\alpha)}$ are defined, as given by expression (A9). The collision frequencies $s^{(\alpha)}$ are given by

$$
s^{(\alpha)} \equiv \frac{5}{3} \sum_{\beta=1}^{N} v^{(\alpha \beta)} \mu^{(\beta)},
$$

where $v^{(\alpha \beta)}$ is defined in (A10). The constants $\mathbf{K}^{(\alpha)}$ are given by

$K_{i}^{(\alpha)} \equiv-\sum_{\beta=1}^{N} v^{(\alpha \beta)} \mu^{(\beta)}[\frac{5}{3}\left(u_{i}^{(\alpha)}-u_{i}^{(\beta)}\right)+\underbrace{\frac{1}{6 \hat{\theta}^{(\alpha \beta)}}\left(\frac{1}{\rho^{(\alpha)}} \hat{h}_{i}^{(\alpha)}-\frac{1}{\rho_{\beta}} \hat{h}_{i}^{(\beta)}\right)}_{(*)}]$.

The term marked as $(*)$ in expressions (25) and (28) is responsible for the prediction of the thermodiffusion effect, as discussed in more detail in Sec. IV C.

The diffusion coefficient $D^{(\alpha)}$ is chosen so that the model predicts the correct evolution of the species temperatures in the continuum limit. This requirement is satisfied, when expression (23) is fulfilled for $X=c_{j}^{(\alpha)} c_{j}^{(\alpha)}$. Applying production terms (A16) and (B11), expression (23) leads to

$$
\begin{aligned}
& -10 \sum_{\beta=1}^{N} v^{(\alpha \beta)} \mu^{(\beta)} \rho^{(\alpha)}\left[\frac{k_{\mathrm{B}}}{m^{(\alpha)}+m^{(\beta)}}\left(T^{(\alpha)}-T^{(\beta)}\right)\right. \\
& \left.-\quad \frac{\mu^{(\beta)}}{3}\left(\mathbf{u}^{(\alpha)}-\mathbf{u}^{(\beta)}\right)^{2}\right] \stackrel{!}{=} 2 m^{(\alpha)}\left\langle A_{i}^{(\alpha)} c_{i}^{(\alpha)} \mid f^{(\alpha)}\right\rangle \\
& \quad+6 \rho^{(\alpha)} D^{(\alpha)} .
\end{aligned}
$$

Using Eq. (26), one can obtain

$$
D^{(\alpha)}=\frac{10}{6} \sum_{\beta=1}^{N} v^{(\alpha \beta)} \mu^{(\beta)} \mu^{(\beta)}\left[\frac{k_{\mathrm{B}} T^{(\alpha)}}{m^{(\alpha)}}+\frac{k_{\mathrm{B}} T^{(\beta)}}{m^{(\beta)}}+\frac{1}{3}\left(\mathbf{u}^{(\alpha)}-\mathbf{u}^{(\beta)}\right)^{2}\right]
$$

for the diffusion coefficient. Note that the diffusion coefficient is always positive, which is an essential requirement for a physical meaningful Fokker-Planck model. 


\section{B. Higher order model}

In this section, a higher order extension for the drift coefficient (26) is constructed, leading the Fokker-Planck model to a correct prediction of species shear stresses and heat fluxes in the continuum limit. Similar to the model of Gorji and Jenny, ${ }^{21}$ the drift coefficient is extended as follows:

$$
A_{i}^{(\alpha)} \equiv-s^{(\alpha)} c_{i}^{(\alpha)}+K_{i}^{(\alpha)}+\underbrace{\psi_{i j}^{(\alpha)} c_{j}^{(\alpha)}+\gamma_{i}^{(\alpha)}\left(c_{j}^{(\alpha)} c_{j}^{(\alpha)}-\left\langle c_{j}^{(\alpha)} c_{j}^{(\alpha)} \mid f^{(\alpha)}\right\rangle\right)}_{N_{i}^{(\alpha)}\left(c_{i}^{(\alpha)}\right)} .
$$

Here, $\psi_{i j}^{(\alpha)}$ denotes a symmetric matrix and $\gamma_{i}^{(\alpha)}$ an vector. Gorji's cubic model (18) additionally contains a third-order parameter to ensure the stability of the model. Such is not included in expression (31), mainly because of the author's experience that the model is stable even without a stabilization parameter. If necessary, however, it would be very easy to extend expression (31) by an additional third-order parameter.

The model parameters $\psi_{i j}^{(\alpha)}$ and $\gamma_{i}^{(\alpha)}$ must be chosen so that identity (23) holds for $X \in\left\{c_{i}^{(\alpha)}, c_{i}^{(\alpha)} c_{i}^{(\alpha)}, c_{<i}^{(\alpha)} c_{j>}^{(\alpha)}, c_{i}^{(\alpha)} c_{j}^{(\alpha)} c_{j}^{(\alpha)}\right\}$. For $X=c_{i}^{(\alpha)}$, model (31) satisfies expression (23), since $\left\langle N_{i}^{(\alpha)} \mid f^{(\alpha)}\right\rangle=0$ and the linear model already fulfills the requirement. Therefore, the model parameters $\psi_{i j}^{(\alpha)}$ and $\gamma_{i}^{(\alpha)}$ are chosen so that

$$
\begin{gathered}
P_{\mathrm{FP}}^{(\alpha)}\left(c_{i}^{(\alpha)} c_{j}^{(\alpha)}\right) \stackrel{!}{=} P_{\mathrm{Boltz}}^{(\alpha)}\left(c_{<i}^{(\alpha)} c_{j>}^{(\alpha)}\right)+\frac{1}{3} \delta_{i j} P_{\mathrm{Boltz}}^{(\alpha)}\left(c_{l}^{(\alpha)} c_{l}^{(\alpha)}\right), \\
P_{\mathrm{FP}}^{(\alpha)}\left(c_{i}^{(\alpha)} \mathbf{c}^{(\alpha)} \mathbf{c}^{(\alpha)}\right) \stackrel{!}{=} P_{\mathrm{Boltz}}^{(\alpha)}\left(c_{i}^{(\alpha)} \mathbf{c}^{(\alpha)} \mathbf{c}^{(\alpha)}\right) .
\end{gathered}
$$

Using $\sum_{i} P_{\text {Boltz }}^{(\alpha)}\left(c_{<i}^{(\alpha)} c_{i>}^{(\alpha)}\right)=0$, it can easily be checked that (32) and (33) lead to a fulfillment of (23) for $X \in\left\{c_{i}^{(\alpha)} c_{i}^{(\alpha)}, c_{<i}^{(\alpha)} c_{j>}^{(\alpha)}\right.$, $\left.c_{i}^{(\alpha)} c_{j}^{(\alpha)} c_{j}^{(\alpha)}\right\}$. Using (32) and (33) and applying the FP production terms (B12) and (B13), a system of nine linear equations for the nine parameters $\psi_{i j}^{(\alpha)}$ and $\gamma_{i}$ can be deduced. The system is discussed in more detail in Appendix $C$.

For the diffusion coefficient, the same expression is applied as in the linear model.

Note that in thermal equilibrium, the right side of the linear system (C2) and (C1) vanishes, which implies that the model parameters $\psi_{i j}^{(\alpha)}$ and $\gamma_{i}^{(\alpha)}$ become zero. Consequently, the higher-order model reduces to the linear model.

\section{Stochastic solution algorithm}

In this section, a stochastic solution algorithm for the model described in Sec. III B is derived. The algorithm is constructed to predict, for the case of a homogeneous gas, a correct evolution of the species temperatures and diffusion velocities, independently of the applied time step size. Consequently, the conservation of energy and momentum is guaranteed. The stochastic equations of motion that are consistent with the higher order model are

$$
\begin{aligned}
& \frac{\mathrm{d} V_{i}^{(\alpha)}}{\mathrm{d} t}=A_{i}^{(\alpha)}+\sqrt{2 D^{(\alpha)}} \frac{\mathrm{d} W_{i}^{(\alpha)}}{\mathrm{d} t} \\
&=-s^{(\alpha)} C_{i}^{\alpha}+K_{i}^{(\alpha)}+N_{i}^{(\alpha)}\left(C_{i}^{\alpha}\right)+\sqrt{2 D^{(\alpha)}} \frac{\mathrm{d} W_{i}^{(\alpha)}}{\mathrm{d} t}, \\
& \frac{\mathrm{d} X_{i}}{\mathrm{~d} t}=V_{i}^{(\alpha)} .
\end{aligned}
$$

Here, $C_{i}^{(\alpha)}=V_{i}^{(\alpha)}-u_{i}^{(\alpha)}$ denotes the thermal part of the velocity $V_{i}^{(\alpha)}$. For the temporal evolution of the species, flow velocity $u_{i}^{(\alpha)}$ follows

$$
\frac{\mathrm{d} u_{i}^{(\alpha)}}{\mathrm{d} t}=\left\langle\frac{\mathrm{d} V_{i}^{(\alpha)}}{\mathrm{d} t}\right\rangle=K_{i}^{(\alpha)}
$$

Combining

$$
\frac{\mathrm{d} V_{i}^{(\alpha)}}{\mathrm{d} t}=\frac{\mathrm{d}\left(C_{i}^{(\alpha)}+u_{i}^{(\alpha)}\right)}{\mathrm{d} t}=\frac{\mathrm{d}\left(C_{i}^{(\alpha)}\right)}{\mathrm{d} t}+K_{i}^{(\alpha)}
$$

with (34) yields a stochastic equation

$$
\frac{\mathrm{d}\left(C_{i}^{(\alpha)}\right)}{\mathrm{d} t}=\underbrace{-s^{(\alpha)} C_{i}^{(\alpha)}+\sqrt{2 D^{(\alpha)}} \frac{\mathrm{d} W_{i}^{(\alpha)}}{\mathrm{d} t}}_{L_{i}^{(\alpha)}}+N_{i}^{(\alpha)}\left(C_{i}^{(\alpha)}\right)
$$

that determinates the thermal particle velocities. In order to solve Eq. (38), a similar approach than described in Ref. 24 is applied. The right side of (38) is divided into a linear part $L_{i}^{(\alpha)}$ and a non-linear part $N_{i}^{(\alpha)}\left(C_{i}^{(\alpha)}\right)$. Assuming constant macroscopic coefficients, for the resulting linear equation

$$
\frac{\mathrm{d}\left(C_{i}^{(\alpha)}\right)}{\mathrm{d} t}=-s^{(\alpha)} C_{i}^{(\alpha)}+\sqrt{2 D^{(\alpha)}} \frac{\mathrm{d} W_{i}^{(\alpha)}}{\mathrm{d} t},
$$

the following analytic solution can be found: ${ }^{3}$

$$
\begin{aligned}
C_{i}^{(\alpha), n+1}= & C_{i}^{(\alpha), n} \exp \left(-s^{(\alpha), n} \Delta t\right) \\
& +\sqrt{\frac{D^{(\alpha), n}}{s^{(\alpha), n}}\left(1-\exp \left(-2 s^{(\alpha), n} \Delta t\right)\right)} \xi_{i}^{(\alpha)} .
\end{aligned}
$$

The indices $n$ and $n+1$ refer to the beginning and the end of the time step, $\Delta t$ denotes the time step size and $\xi_{i}^{(\alpha)}$ are independent standard normal variates. For the non-linear part $N_{i}^{(\alpha)}\left(C_{i}^{(\alpha)}\right)$, a simple Euler scheme is applied. In summary,

$$
\begin{aligned}
C_{i}^{(\alpha), n+1}= & C_{i}^{(\alpha), n} \exp \left(-s^{(\alpha), n} \Delta t\right)+\frac{N_{i}^{(\alpha)}\left(C_{i}^{(\alpha), n}\right)}{s^{(\alpha), n}}\left(1-\exp \left(s^{(\alpha), n} \Delta t\right)\right) \\
& +\sqrt{\frac{D^{(\alpha), n}}{s^{(\alpha), n}}\left(1-\exp \left(-2 s^{(\alpha), n} \Delta t\right)\right)} \xi_{i}^{(\alpha)}
\end{aligned}
$$

can be found as a solution for (38). The Euler integration of the non-linear part leads to a wrong prediction of the species temperatures. This error can be corrected by the following scaling of the new particle velocities: 


$$
\mathbf{C}^{(\alpha), n+1} \rightarrow \epsilon \cdot \mathbf{C}^{(\alpha), n+1} .
$$

The scaling parameter $\epsilon$ is defined as

$$
\epsilon=\sqrt{\frac{3 k_{\mathrm{B}} T^{(\alpha), n+1} / m^{(\alpha)}}{\left\langle C_{i}^{(\alpha), n+1} C_{i}^{(\alpha), n+1} \mid f^{(\alpha)}\right\rangle / n^{(\alpha)}}},
$$

where $T^{(\alpha), n+1}$ denotes the correct species temperatures at the end of the time step. The temperatures $T^{(\alpha), n+1}$ are obtained by solving a system of differential equations that is discussed in more detail in Appendix D. The final particle velocities are calculated as

$$
\mathbf{V}^{(\alpha), n+1}=\epsilon \cdot \mathbf{C}^{(\alpha), n+1}+\mathbf{u}^{(\alpha), n+1} .
$$

The species flow velocities $u_{i}^{(\alpha), n+1}$ are also obtained as a solution of the system of differential equations, described in Appendix D.

As described by Eq. (44), the final particle velocities are scaled and shifted to adopt the species temperatures $T^{(\alpha), n+1}$ and flow velocities $u_{i}^{(\alpha), n+1}$ at the end of the time step. These quantities are calculated based on the system of differential equations, discussed in more detail in Appendix D. This system captures the time evolution of macroscopic quantities for a homogeneous gas. As long as the system is properly integrated, Eq. (44), therefore, leads to correct species temperatures and flow velocities in a homogeneous flow, regardless of the time step size.

For the calculation of the particle positions, Eq. (35) has to be integrated. For the sake of simplicity, the positions

$$
\mathbf{X}^{(\alpha), n+1}=\mathbf{V}^{(\alpha), n} \Delta t+\mathbf{X}^{(\alpha), n}
$$

are updated by assuming free flight trajectories. In summary, particle positions and velocities are updated as described in Algorithm 1. Due to the simple position integration scheme (45), the time step should not be much larger than the local mean collision time to avoid errors caused by numerical diffusion. However, this limitation could be avoided by using more accurate but also more complex integration schemes.

\section{Averaging of moments}

The correct evaluation of velocity moments is a crucial task for the execution of the particle algorithm described in Algorithm 1. In the simplest case, a moment

$$
\frac{1}{n^{(\alpha)}}\left\langle Q^{(\alpha)} \mid f^{(\alpha)}\right\rangle \approx \frac{1}{N_{\mathrm{p}}} \sum_{i=1}^{N_{\mathrm{p}}} Q_{i}^{(\alpha)}
$$

\section{ALGORITHM 1. Calculation of new particle positions and velocities.}

1. Evaluate the required statistical moments for every grid cell

2. Solve systems (C1) and (C2) to obtain the model parameters $\psi_{i j}^{(\alpha)}$ and $\gamma_{i}^{(\alpha)}$ for every grid cell

3. Calculate the new thermal particle velocities using Eq. (41)

4. Solve system (D1) for every grid cell, applying $\left(\mathbf{u}^{(\alpha), n}, T^{(\alpha), n}\right.$, $\left.\mathbf{q}^{(\alpha), n}\right)$ as initial condition

5. Calculate the final particle velocities using Eq. (44)

6. Calculate the final particle positions using Eq. (45) can be approximated by instantaneously averaging over all particles of a grid cell. Here, $N_{\mathrm{p}}$ means the number of $\alpha$ particles in the grid cell and $f^{(\alpha)}$ denotes the distribution function, the particles are representing. However, for a finite number of particles, expression (46) can lead to additional bias errors. ${ }^{37}$ To reduce these errors, an underrelaxation technique can be applied for stationary problems. ${ }^{13}$ For this purpose, a average number of particles per cell and species $X^{(\alpha), n}$ at time step $n$ is calculated by

$$
X^{(\alpha), n+1} \equiv \mu X^{(\alpha), n}+(1-\mu) N_{\mathrm{p}}^{(\alpha), n+1},
$$

where $\mu \in(0,1)$ denotes a weighting factor. The index $n$ refers to the time step. Similarly, time averaged moments

$$
Y^{(\alpha), n+1} \equiv \mu Y^{(\alpha), n}+(1-\mu) \sum_{i=1}^{N_{\mathrm{p}}^{(\alpha), n+1}} Q_{i}^{(\alpha)}
$$

are continuously calculated for each time step. Finally, statistical moments at time step $n$ can be calculated as

$$
\frac{1}{n}\left\langle Q^{(\alpha), n+1} \mid f^{(\alpha)}\right\rangle \approx \frac{Y^{(\alpha), n+1}}{X^{(\alpha), n+1}} .
$$

Using $\mu=1-\frac{1}{N_{\text {ave }}}$ leads to similar statistical noise than regular time averaging over $N_{\text {ave }}$ time steps. Time averaged moments as defined by (49) always develop slower than the instantaneous averaged moments as given by expression (46). Therefore, the use of time averaged moments in unsteady problems is not recommended. However, for steady gas flows featuring a small signal to noise ratio, this technique can dramatically reduce the number of required particles per cell.

\section{Test cases}

In this section, the proposed model is applied to various test cases to discuss its performance and efficiency for describing nonequilibrium gas flows. A He-Ar mixture is used for all simulations. The molecular reference diameters are listed in Table. I.

The mixture Knudsen number is defined by

$$
K n \equiv \frac{\lambda_{\mathrm{mix}}}{l} .
$$

Here, $l$ denotes a reference length scale and the average mixture mean free path is given by

$$
\lambda_{\text {mix }}=\sum_{\alpha=1}^{N} \frac{n^{(\alpha)}}{n} \lambda^{(\alpha)},
$$

TABLE I. HS collision parameter. ${ }^{6}$

\begin{tabular}{llc}
\hline \hline Species $\alpha$ & Species $\beta$ & $d^{(\alpha \beta)}\left(10^{-10} \mathrm{~m}\right)$ \\
\hline $\mathrm{He}$ & $\mathrm{He}$ & 2.33 \\
$\mathrm{He}$ & $\mathrm{Ar}$ & 3.25 \\
$\mathrm{Ar}$ & $\mathrm{Ar}$ & 4.17 \\
\hline \hline
\end{tabular}


where $n=\sum_{\alpha=1}^{N} n^{(\alpha)}$ denotes the mixture number density and

$$
\lambda^{(\alpha)} \equiv\left[\sum_{\beta=1}^{N} \pi\left(d^{(\alpha-\beta)}\right)^{2} n^{(\beta)} \sqrt{1+\frac{m^{(\beta)}}{m^{(\alpha)}}}\right]^{-1}
$$

the mean free path of a species $\alpha$ particle. The mole fraction $\chi^{(\alpha)}$ is defined as

$$
\chi^{(\alpha)} \equiv \frac{n^{(\alpha)}}{n} .
$$

For all test cases, reference DSMC simulations assuming the hardsphere collision model are performed. To avoid different results due to numerical discretization, the same spatial and temporal discretization is used for DSMC and Fokker-Planck simulations. All simulations are performed with the SPARTA code, ${ }^{39-42}$ which is extended by the Fokker-Planck model proposed in this work.

\section{A. Heat bath}

The homogeneous relaxation of species diffusion velocities and temperatures in a heat bath is studied. To investigate the relaxation process of diffusion velocities, particle velocities are set at the beginning of the simulation according to a Maxwell distribution with a temperature of $T_{\text {mix }}=300 \mathrm{~K}$. The distribution of He velocities is additional shifted by $v_{0}^{(\mathrm{He})}=180 \mathrm{~m} / \mathrm{s}$ in the $x$ direction. To investigate the relaxation process of the species temperatures, the particle velocities are set at the beginning of the simulation according to a Maxwell distribution with temperature $T_{0}^{(\mathrm{He})}=600 \mathrm{~K}$ for the $\mathrm{He}$ particles and temperature $T_{0}^{(\mathrm{Ar})}=300 \mathrm{~K}$ for the Ar particles. In both cases, number densities of $n^{(\mathrm{Ar})}=2 \times 10^{20} 1 / \mathrm{m}^{3}$ and $n^{(\mathrm{He})}$ $=10 \times 10^{20} 1 / \mathrm{m}^{3}$ are used. For each simulation, a single grid cell with total 5000 particles is simulated. Since both relaxation processes are non-stationary problems, the results of 100 calculations are averaged for each case.

Figure 2 shows the relaxation process of the species flow velocities and temperatures. The simulation time $t$ is normalized to an average collision time $\tau=1 / v$, with $v$ defined as

$$
\begin{aligned}
v & \equiv \frac{1}{2}\left[v^{(\mathrm{He}-\mathrm{Ar})}(T=300 \mathrm{~K})+v^{(\mathrm{Ar}-\mathrm{He})}(T=300 \mathrm{~K})\right] \\
& =\frac{16}{5} \sqrt{\pi} d^{(\mathrm{Ar}-\mathrm{He}) 2} \sqrt{\frac{k_{\mathrm{B}} 300 \mathrm{~K}}{m^{(\mathrm{He})}}+\frac{k_{\mathrm{B}} 300 \mathrm{~K}}{m^{(\mathrm{Ar})}}} n_{\text {mix }}
\end{aligned}
$$

and $n_{\text {mix }}=n^{(\mathrm{He})}+n^{(\mathrm{Ar})}$. The equilibrium values

$$
v_{\text {eq }}=\frac{\rho^{(\mathrm{He})} v^{(\mathrm{He})}}{\rho^{(\mathrm{He})}+\rho^{(\mathrm{Ar})}}=60 \frac{\mathrm{m}}{\mathrm{s}},
$$

$$
T_{\mathrm{eq}}=\frac{n^{(\mathrm{He})} T^{(\mathrm{He})}+n^{(\mathrm{Ar})} T^{(\mathrm{Ar})}}{n^{(\mathrm{He})}+n^{(\mathrm{Ar})}}=550 \mathrm{~K}
$$

at the end of the relaxation process are correctly predicted by the Fokker-Planck model. A good agreement between FokkerPlanck and DSMC results during the relaxation process can also be observed.

Figure 3 shows four different energy distribution functions along the relaxation process of the species temperatures. Times at which the distribution functions are calculated are marked on the right side of Fig. 2. Again, a good agreement between FokkerPlanck and DSMC results can be observed. The equilibrium distribution $f_{4}^{(\alpha)}$ at the end of the relaxation process as well as the non-equilibrium distributions $f_{1}^{(\alpha)}, f_{2}^{(\alpha)}$, and $f_{3}^{(\alpha)}$ along the relaxation process is correctly predicted by the Fokker-Planck model.

Figure 4 shows two different particle velocity distribution functions at the beginning and at the end of the relaxation process of the species velocities. The particle velocity is normalized to the most probable speed $c^{*(\alpha)}=\sqrt{2 \frac{k_{\mathrm{B}} 300 \mathrm{~K}}{m^{(\alpha)}}}$. Times at which the distributions are calculated are also marked on the left side of Fig. 2. Again, a good agreement between Fokker-Planck and DSMC results can be observed.

In summary, the good agreement between FP and DSMC results is somewhat unexpected since the FP model is only valid for near continuum flows. However, the good agreement to DSMC results is discussed in more detail in Sec. IV E.

\section{B. Couette flow}

A Couette flow is investigated. The flow is modeled in three dimensions. Domain boundaries in the $x$-direction are modeled as fully diffusive walls with a temperature of $T_{\mathrm{w}}=300 \mathrm{~K}$. The lower wall is stationary, while the higher wall moves in the $y$-direction with a velocity of $v_{\mathrm{w}}=300 \mathrm{~m} / \mathrm{s}$. The distance between both walls is set to
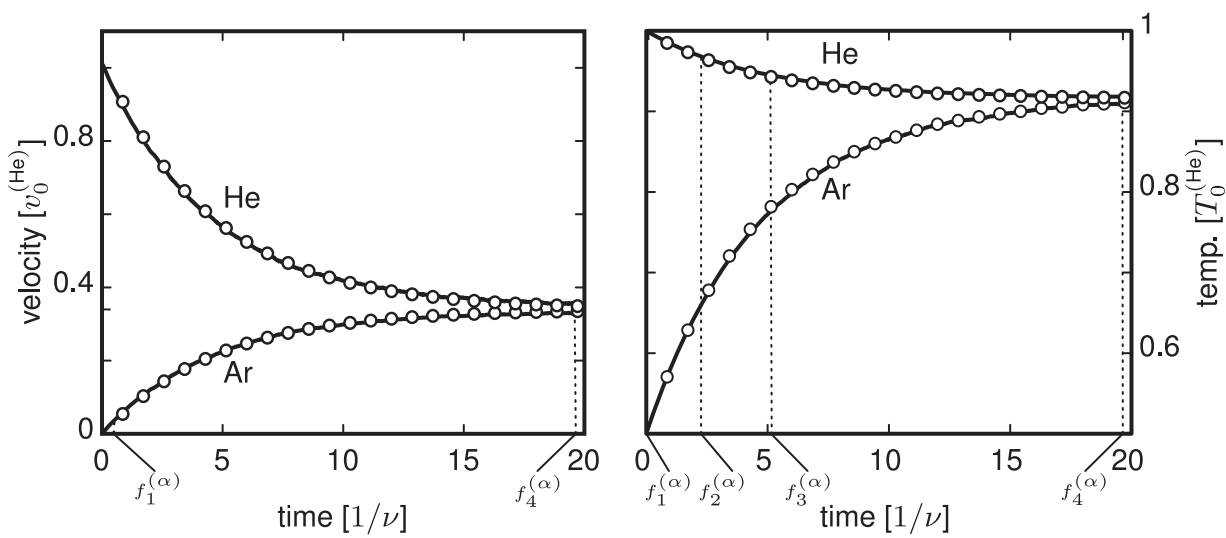

FIG. 2. Left: relaxation of species flow velocities in terms of the initial He velocity. Right: relaxation of species temperatures in terms of the initial $\mathrm{He}$ temperature. Lines: DSMC results and circles: FP results. 

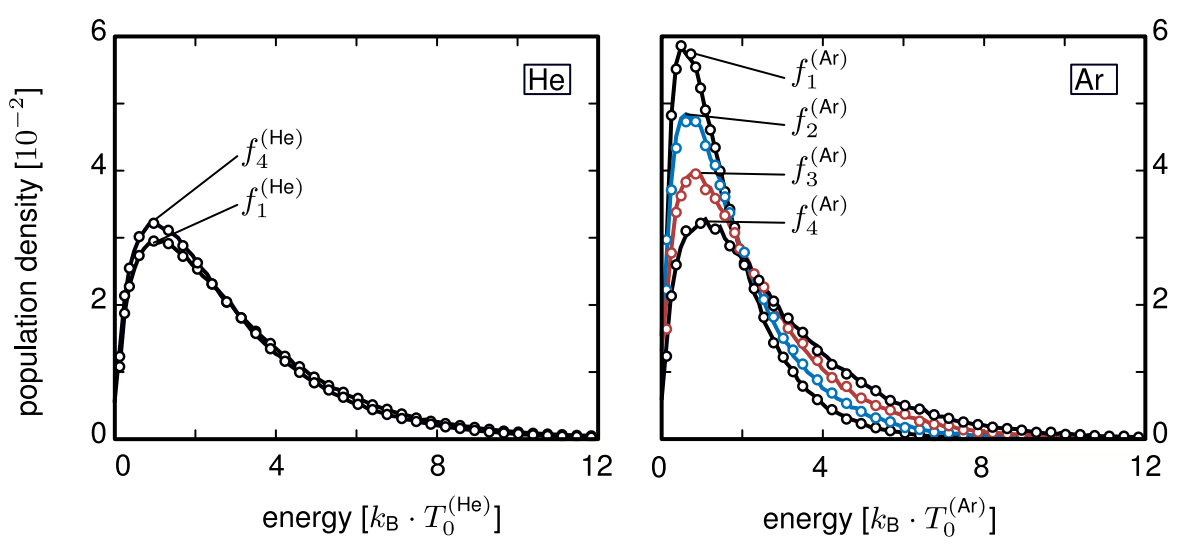

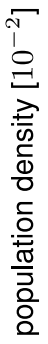

FIG. 3. Left: relaxation of the energy distribution function for He particles. Right: relaxation of the energy distribution function for Ar particles. Lines: DSMC results and circles: FP results.
$1 \mathrm{~m}$, which is also used as a reference length for defining the Knudsen number (50). The domain boundaries in the $y$ - and $z$-direction are set as periodic. Simulations are performed for different Knudsen numbers $K n \in\{1,0.5,0.25,0.05\}$ assuming a constant mole fraction $\chi^{(\mathrm{He})}=0.5$ and for different mole fractions $\chi^{(\mathrm{He})} \in\{0.25,0.5,0.75\}$ assuming a constant Knudsen number $K n=0.05$. The domain is divided into 100 equal sized cells in the $\mathrm{x}$-direction, resulting in a cell size $\Delta x$, which is seven times smaller than the mean free path $\lambda^{(\mathrm{He})}$ and three times smaller than $\lambda^{(\mathrm{Ar})}$ for the smallest Knudsen number case. The time step size is set to $\Delta t=\frac{\Delta x}{5 \cdot c^{*}(\mathrm{He})+300 \frac{\mathrm{m}}{\mathrm{s}}} \approx 2 \cdot 10^{-6} \mathrm{~s}$, where $c^{*(\mathrm{He})}=\sqrt{2 \frac{k_{\mathrm{B}} 300 \mathrm{~K}}{m^{(\mathrm{He})}}}$ denotes the most probable speed of a He particle. To reduce the amount of statistical fluctuations, the scaling factor $f_{\mathrm{N}}$ between the number of real and simulated molecules is set to obtain a minimum number of 200 particles per cell and per species. A steady flow is achieved after 50000 simulation time steps. Afterwards, the under-relaxation technique as described in Sec. III D is applied, using a weighting factor of $v=0.001$. To obtain the final results, averages are taken every ten time steps to the total number of 90000 averages, with exception of the case with the lowest Knudsen number, where the averages are taken every 100 time steps.

Figure 5 shows the species flow velocities along the simulation domain for cases with different Knudsen numbers. For the case with the lowest Knudsen number, the shape of the velocity distribution is linear, both species adopt similar flow velocities and slip effects at the walls are negligible small. As the Knudsen number increases, slip effects become dominant and the velocity distribution adopts a non-linear shape. For the case with the largest Knudsen, the species adopt different flow velocities in the direct vicinity of the walls. The Fokker-Planck model accurately predicts velocity slips on the walls as well as the non-linear shape of the velocity distribution with increasing Knudsen number. Differences between species velocities for the large Knudsen number case are, in principle, predicted.

Figure 6 shows the shear stresses of $\mathrm{Ar}$ and $\mathrm{He}$ for cases with different Knudsen numbers. The large variance in results for the lowest Knudsen case may be due to non-independent samples used for averaging. For the case with the lowest Knudsen number, a good agreement is found between Fokker-Planck and DSMC results, whereas in the higher Knudsen number cases, the FokkerPlanck model slightly over predicts the shear stresses compared to the DSMC simulations. The deviations are larger for the He species, which might be due to the large mass ratio $\frac{m^{(\mathrm{Ar})}}{m^{(\mathrm{He})}} \approx 10$, causing the He species to reach thermal non-equilibrium faster.

Figure 7 shows species shear stresses for cases with different mole fractions. Independently of the mixture composition, shear stresses are accurately predicted by the Fokker-Planck model.
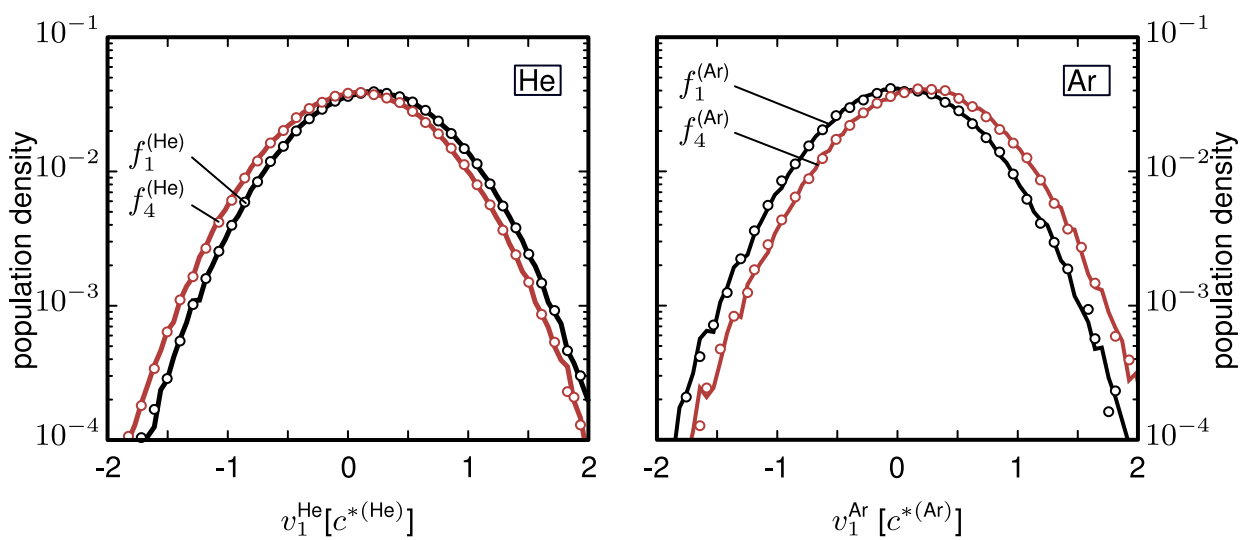

FIG. 4. Left: relaxation of the velocity distribution function for $\mathrm{He}$ particles. Right: relaxation of the velocity distribution function for Ar particles. Lines: DSMC results and circles: FP results. 

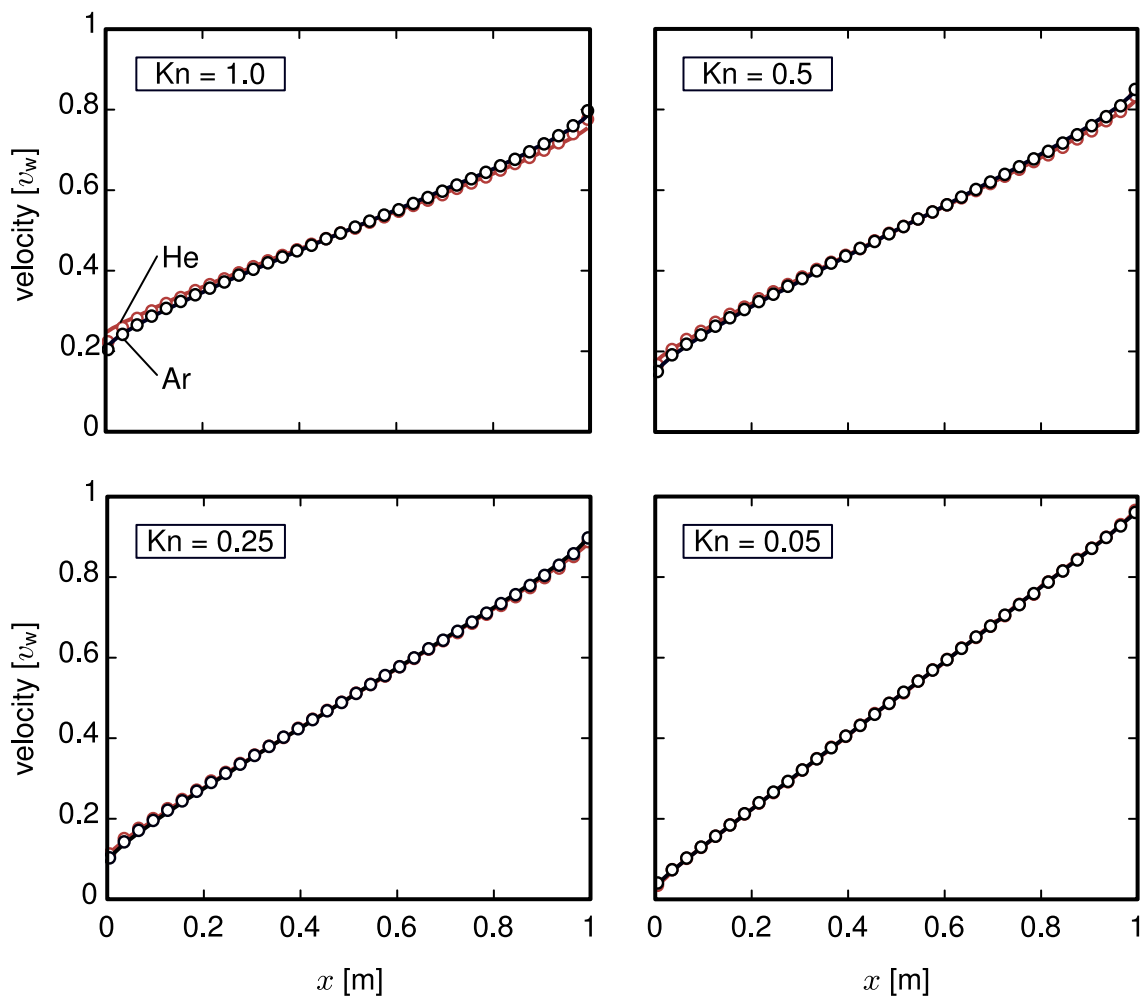

FIG. 5. Flow velocity of $\mathrm{Ar}$ and $\mathrm{He}$ in terms of the wall velocity $v_{\mathrm{w}}$ for different Knudsen numbers. Lines: DSMC results and circles: FP results. Black: Ar and red: $\mathrm{He}$.

The reader should note that the correct prediction of shear stresses and flow velocities suggests a correct prediction of the mixture viscosity in the continuum limit.

\section{Supersonic Couette flow}

The supersonic Couette flow is investigated. In contrast to the subsonic Couette flow studied in Sec. IV B, the supersonic Couette flow features strong heat fluxes that allow us to study phenomena, such as thermodiffusion.

The same simulation domain and spatial resolution as described in Sec. IV B for the subsonic Couette flow is used, only the velocity of the moving wall is changed to $v_{\mathrm{w}}=1000 \mathrm{~m} / \mathrm{s}$.
Simulations are performed for a Knudsen number of $K n=0.05$ and three different global mole fractions $\chi^{(\mathrm{He})} \in\{0.25,0.5,0.75\}$. Later results show that the species temperatures increase up to $T_{\max }$ $\approx 450 \mathrm{~K}$ along the simulation domain. Therefore, the time step size is adjusted to $\Delta t=\frac{\Delta x}{5 \cdot c^{*(\mathrm{He})}+1000 \frac{\mathrm{m}}{\mathrm{s}}} \approx 1 \times 10^{-6} \mathrm{~s}$ with $c^{*(\mathrm{He})}=\sqrt{2 \frac{k_{\mathrm{B}} T_{\max }}{m^{(\mathrm{He})}}}$. The scaling factor $f_{\mathrm{N}}$ is set to obtain a minimum number of 200 particles per cell and per species. A steady flow is assumed after 50000 simulation steps. Because of the large signal to noise ratio, no underrelaxation technique needs to be applied. To obtain the final result, averages are taken every 20 time steps up to the total number of 90000 averages.
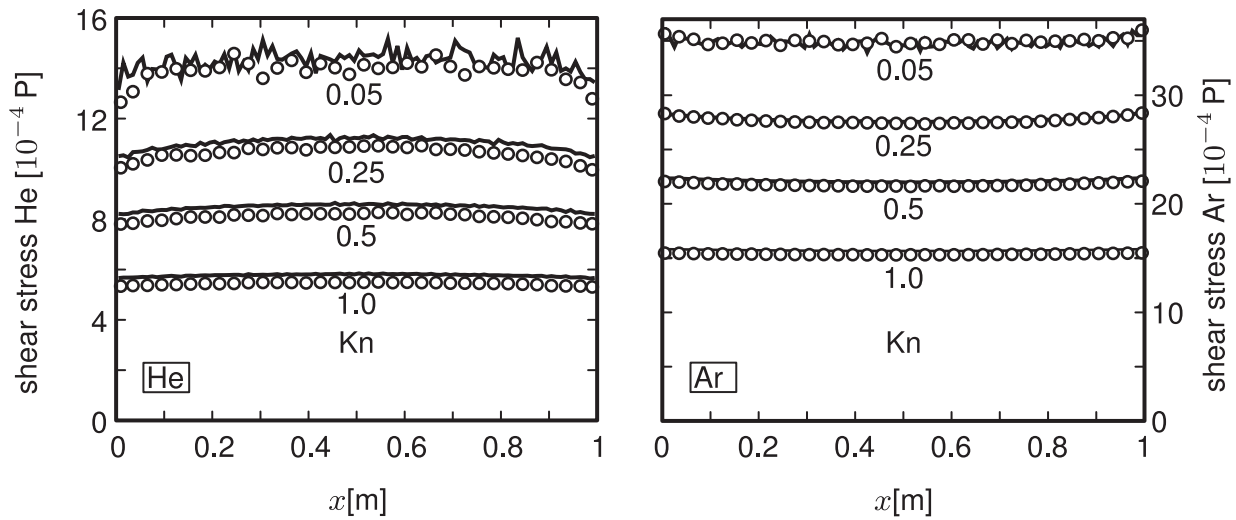

FIG. 6. Left: shear stress of He. Right: shear stress of Ar. Lines: DSMC results and circles: FP results. 


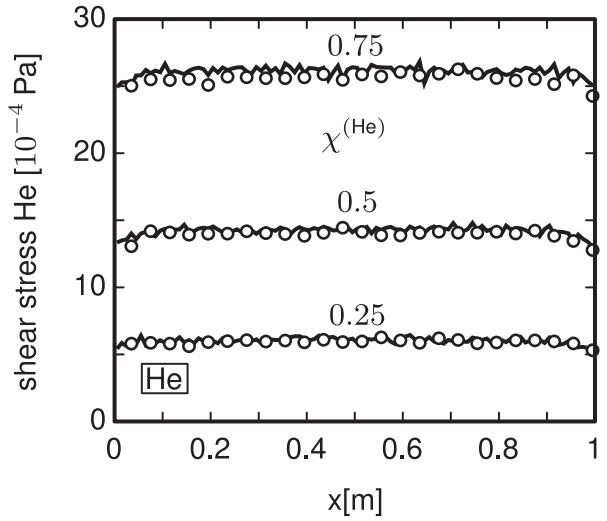

The left upper picture in Fig. 8 shows the mixture flow velocity for the case with a mole fraction of $\chi^{(\mathrm{He})}=0.5$. As for the Couette flow, the velocity distribution is linear, slip effects are negligible, and flow velocities are similar for different species. Again, FokkerPlanck results fit very well with the DSMC results. The remaining pictures in Fig. 8 show density distributions along the simulation domain. The parabolic shape of the distributions is due to the parabolic shape of the temperature distributions (see the right side of Fig. 9) and a constant pressure along the simulation domain. A good agreement between Fokker-Planck and DSMC results can be detected. Worth noting is the separation of the species densities for the case with a mole fraction $\chi^{(\mathrm{He})}=0.5$. Since the binary diffusion coefficient is symmetric, ${ }^{34}$ this separation cannot be caused by classical mass or pressure diffusion. Instead, the separation must be the result of thermodiffusion. ${ }^{6}$ As described by Burgers, ${ }^{26}$ thermodiffusion is induced by interaction of heat fluxes in production terms $P^{(\alpha)}\left(c_{i}^{(\alpha)} c_{j}^{(\alpha)} c_{j}^{(\alpha)}\right)$, as given, for example, by the term marked as $(*)$ in expression (28). To further study this effect, Fokker-Planck calculations without thermodiffusion are performed by simply neglecting this term. The left side of Fig. 9 shows the resulting density distributions for the case with a mole fraction of $\chi^{(\mathrm{He})}=0.5$. While the standard FP simulation accurately predicts the species separation, the FP simulation with the neglected term does not predict species separation. This results show that thermal diffusion can explicitly turned on or off for the FP model, simply by including or excluding the term marked by $(*)$ in expression (28).
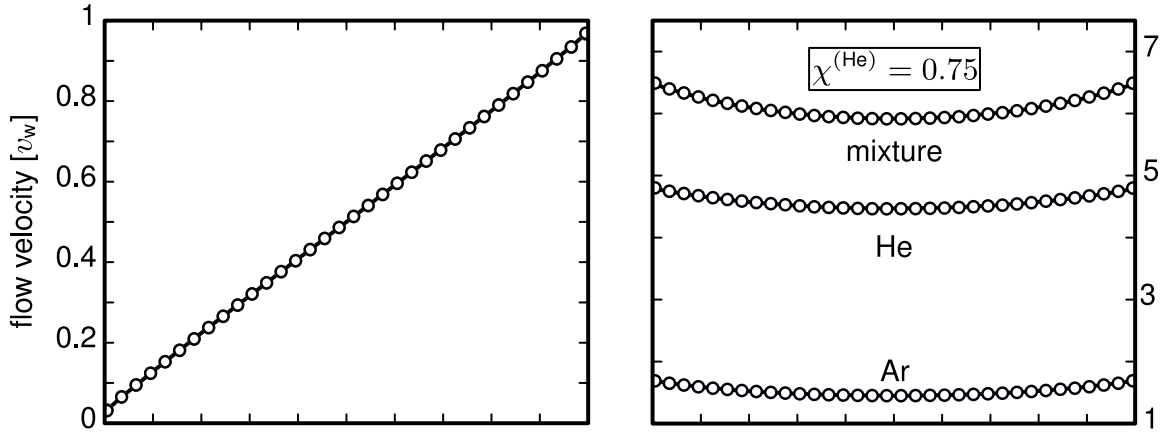

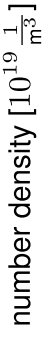

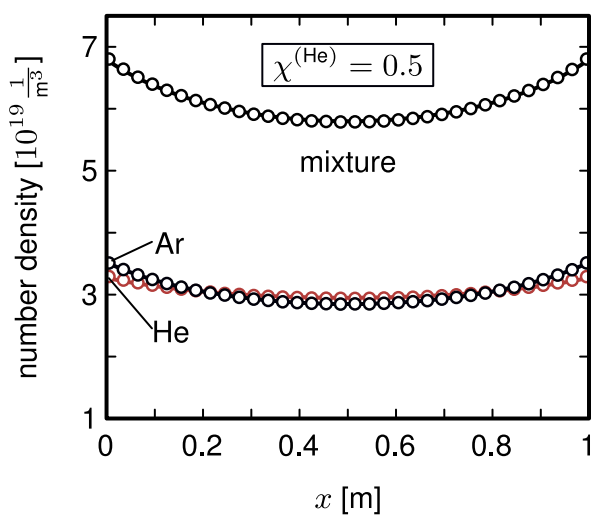

FIG. 8. Left upper picture: mixture flow velocity in terms of the wall velocity $v_{\mathrm{w}}$ for a density ratio of $\chi^{(\mathrm{He})}=0.5$. Other pictures: density distributions for different mole fractions. Lines: DSMC results and circles: FP results. 

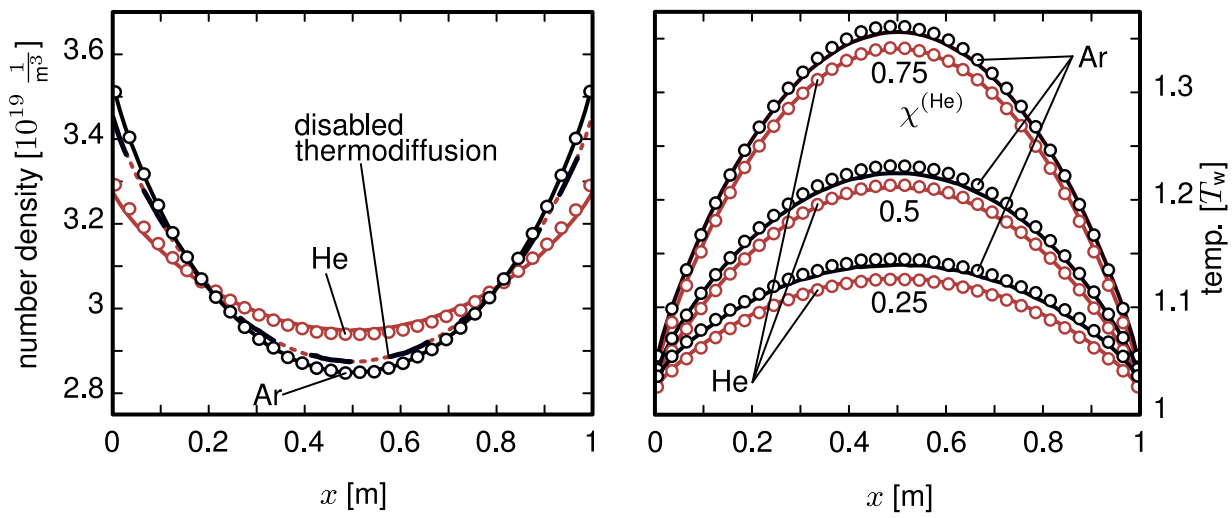

FIG. 9. Left: species density distributions of $\mathrm{Ar}$ and $\mathrm{He}$ for a mole fraction of $\chi^{(\mathrm{He})}$ $=0.5$. Right: species temperature distributions of $\mathrm{Ar}$ and $\mathrm{He}$ for different mole fractions. Lines: DSMC results and circles: FP results.
The right side of Fig. 9 shows the temperature distributions along the simulation domain. The temperature increases with an increasing $\mathrm{He}$ fraction due to the growth of the dissipated flow energy. Species temperatures are separated, and a significant temperature slip occurs at the walls. With exception of small deviations for the Ar species, the Fokker-Planck model accurately reproduced DSMC results. Figure 10 shows the species shear stresses. The shear stresses are not constant over the domain, indicating a strong degree of thermal non-equilibrium. However, the Fokker-Planck model accurately predicts the shear stress distributions expect of small deviations for the case with a mole fraction of $\chi^{(\mathrm{He})}=0.75$.

Figure 11 shows the distributions of the species heat fluxes. The distributions feature a non-linear linear shape, indicating again a strong degree of thermal non-equilibrium. Again, the Fokker-Planck model accurately reproduces DSMC results.

\section{One-dimensional mass diffusion}

A one-dimensional diffusion test case is investigated. The flow is described three-dimensionally. The lower $x$-boundary is modeled as a reservoir of Ar particles and the higher $x$-boundary as a reservoir of He particles. For both reservoirs, the same temperature $T_{0}=300 \mathrm{~K}$ is set. The distance between the $\mathrm{x}$-boundaries is set to $1 \mathrm{~m}$, which is also used as a reference length to define the Knudsen number (50). For the $\mathrm{y}$ - and $\mathrm{z}$-boundaries, periodic boundary conditions are applied. Reservoir densities are set to achieve different Knudsen numbers $K n \in\{0.5,0.25,0.1,0.05\}$ and a constant density fraction $\chi^{(\mathrm{He})}=0.5$. The domain is divided into 100 equal sized cells in the $\mathrm{x}$-direction resulting in a cell size $\Delta x$, which is seven times smaller than the mean free path $\lambda^{(\mathrm{He})}$ and three times smaller than $\lambda^{(\mathrm{Ar})}$ for the smallest Knudsen number case. Later simulations show that the He flow velocity increases up to $800 \frac{\mathrm{m}}{\mathrm{s}}$ in the $\mathrm{x}$-direction. Therefore, the time step size is set to $\Delta t=\frac{\Delta x}{5 \cdot c^{*}(\mathrm{He})+800 \frac{\mathrm{m}}{\mathrm{s}}}$ $\approx 2 \times 10^{-6} \frac{\mathrm{m}}{\mathrm{s}}$ with $c^{*(\mathrm{He})}=\sqrt{2 \frac{k_{\mathrm{B}} 300 \mathrm{~K}}{\mathrm{~m}^{(\mathrm{He})}}}$. The number of simulated molecules is adjusted to obtain the minimum number of 15 particles per cell and per species. The flow is assumed to be steady after 50000 time steps. Because of the large signal to noise ratio, no under-relaxation technique is applied. To obtain the final results, averages are taken every ten time steps up to the total number of 90000 averages.

Figure 12 shows the distribution of the species densities, normalized to the reservoir densities. For the flow with the lowest Knudsen number, a good agreement between Fokker-Planck and DSMC results can be found. As the Knudsen number increases, increasing differences can be detected. Large deviations can be found, in particular, for the Ar species.

Figure 13 shows the distribution of the species flow velocities. Even for the case with the lowest Knudsen number, small deviations between the Fokker-Planck and DSMC can be detected. The reason
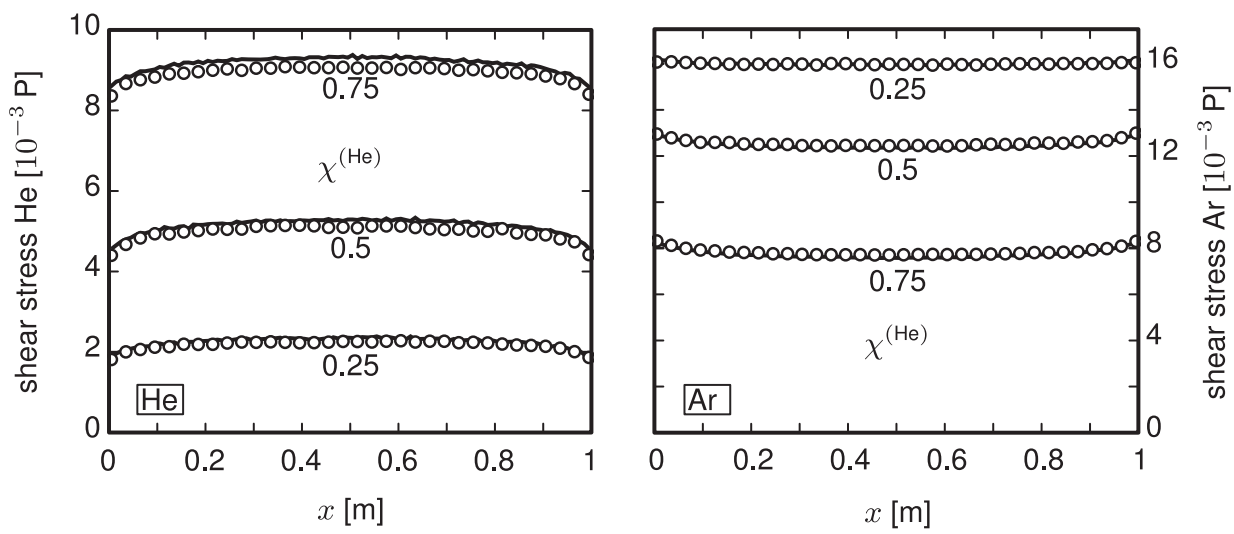

FIG. 10. Left: shear stress of He for different mole fractions. Right: shear stress of $\mathrm{Ar}$ for different mole fractions. Lines: DSMC results and circles: FP results. 

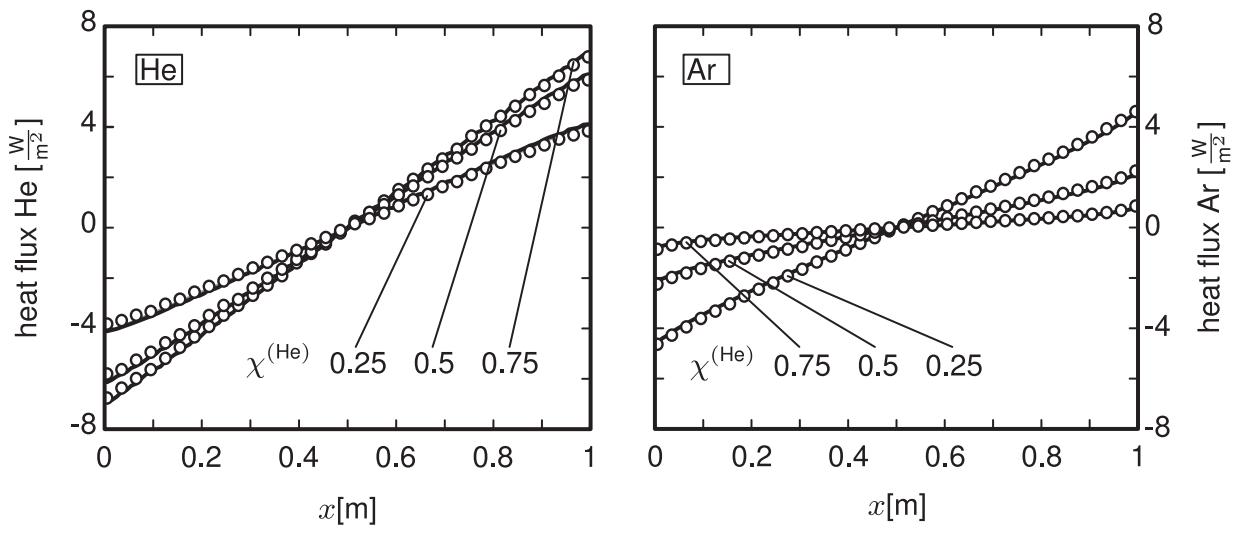

FIG. 11. Left: heat flux of He for different mole fractions. Right: heat flux of $\mathrm{Ar}$ for different mole fractions. Lines: DSMC results and circles: FP results. for this disagreement can be explained by the production terms that have been utilized to construct the FP model. The terms (A1)-(A4) are of linear order. This approximation is only valid if differences between the species flow velocities are small, which is clearly not the case for the simulations in this section, even for the flow with the smallest Knudsen number. As the Knudsen number increases, differences between DSMC and Fokker-Planck results become more dominant.

\section{E. Performance considerations}

This section attempts to evaluate the performance of the FP model to describe non-equilibrium flows based on the previously examined test cases. In general, the model shows a good performance for describing non-equilibrium flows. Even for strong non-equilibrium flows, no major deviations to reference DSMC calculations can be detected. Upon closer inspection, the following conclusions can be drawn.

The performance of the model depends on the quantity that is investigated. For example, the FP model accurately predicts the flow velocity for the Couette flow even for high Knudsen numbers. In contrast, deviations can be observed for the shear stresses. The FP model also accurately reproduces density distributions for the supersonic Couette flow, while small deviations can be observed again for the shear stress. This observation could be generalized to the statement that the performance of the model increases for lower order velocity moments.
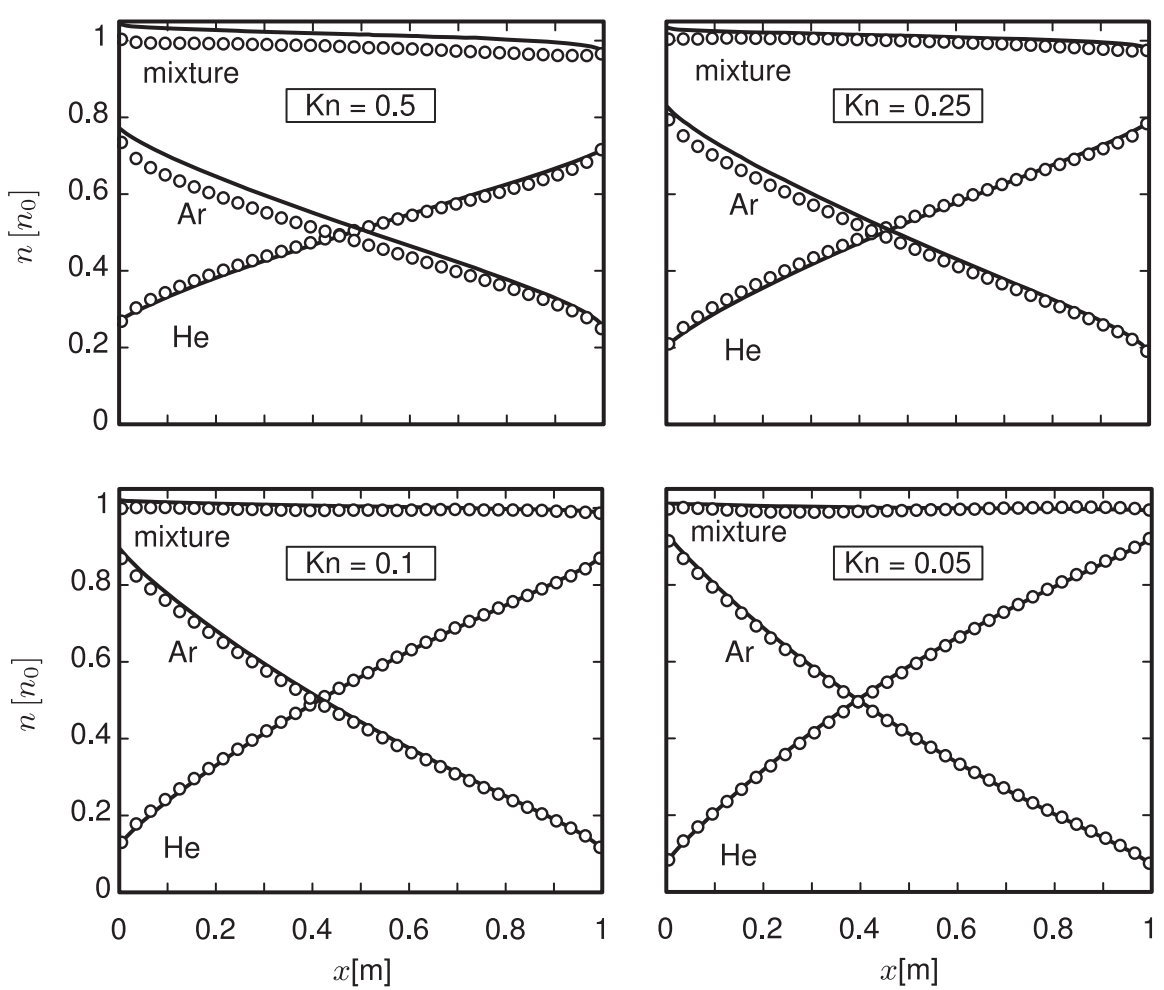

FIG. 12. Number densities of $\mathrm{He}, \mathrm{Ar}$, and the entire mixture in terms of the reservoir densities $n_{0}$ for different Knudsen numbers. Lines: DSMC results and circles: FP results. 

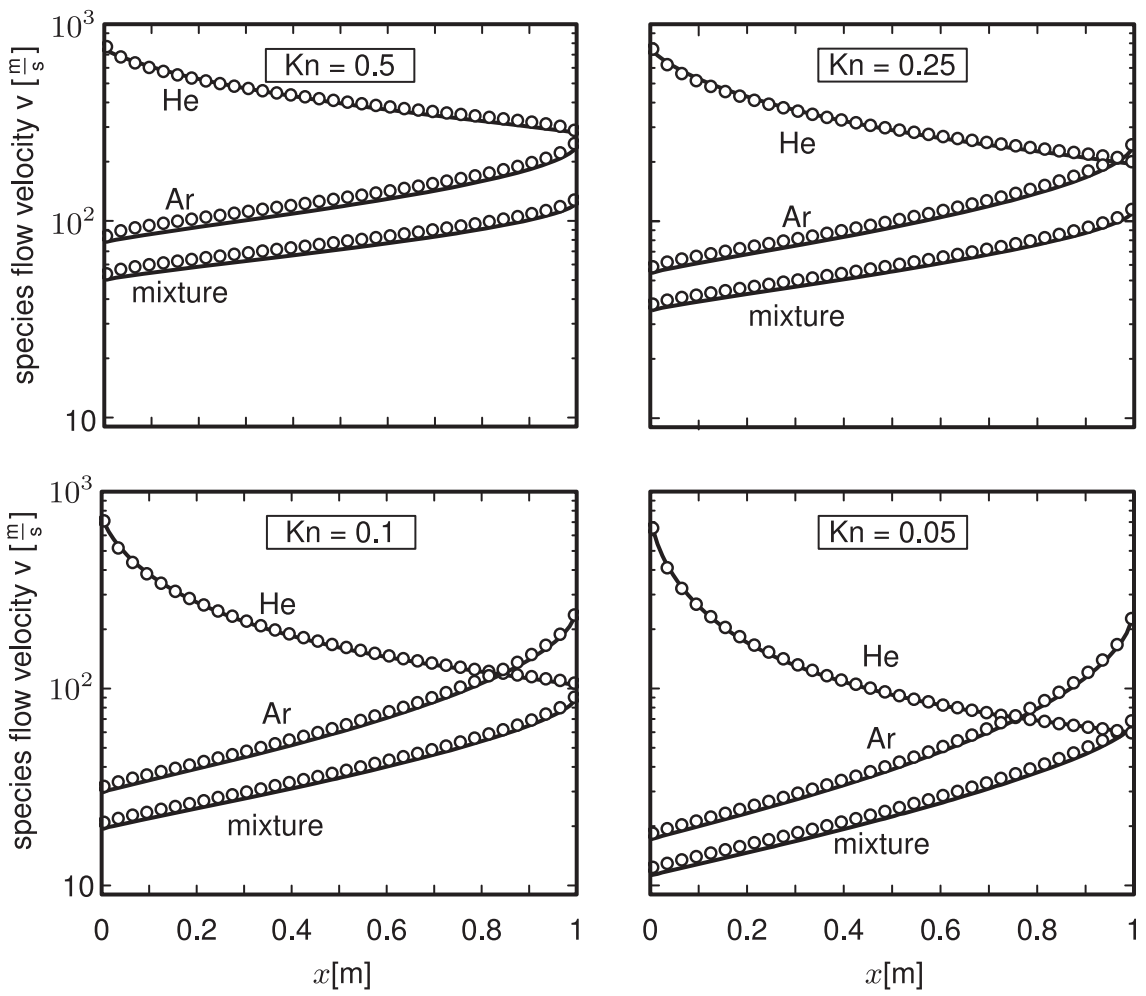

FIG. 13. Magnitude of the flow velocity for $\mathrm{Ar}$, $\mathrm{He}$, and the entire mixture for different Knudsen numbers. Lines: DSMC results and circles: FP results.

The model shows a good performance with varying Knudsen number. As expected, the deviations from reference DSMC simulations become larger with increasing Knudsen numbers, but important non-equilibrium effects, for example, slip effects for the Couette flow are accurately predicted.

The FP model is able to model flows in which different species assume different flow states, such as in the diffusion test case, in which species assume very different flow velocities and in the relaxation test case, where species adopt different temperatures. This can be explained by the modeling approach made that assumes for each species a separate distribution function and Fokker-Planck equation.

The model is able to handle the mixture that features species with a large mass ratio. For example, all simulations are performed for an He-Ar mixture, featuring a mass fraction of $m^{(\mathrm{Ar})} / m^{(\mathrm{Ar})} \approx 10$.

Finally, the reader should remember that this conclusion is based only on very simple test cases. Additional investigations are required to examine the performance of the model for realistic scenarios.

\section{F. Computational efficiency}

The main motivation for the introduction of the FP model is to reduce the computational effort in comparison to conventional particle simulation methods, such as DSMC. Hence, in order to evaluate the efficiency of our model, the computing effort of a pure DSMC simulation is compared with the effort of our FP model. In general, such a comparison is a complex task, especially since the DSMC method must resolve molecular scales, while the FP algorithm does not. Hence, a satisfactory efficiency study would require DSMC and FP simulations with different temporal and spatial resolutions. Such a detailed study would go beyond the scope of this paper. Instead, we examine the scaling of the computing time with respect to the Knudsen number.

For this, the heat bath test case, as described in Sec. IV A, is studied. Calculations are performed for an $\mathrm{He}-\mathrm{Ar}$ mixture with equalized temperatures $T_{0}^{(\mathrm{He})}=T_{0}^{(\mathrm{Ar})}$ and vanishing flow velocities. Figure 14 shows the relative computing time of DSMC and FP

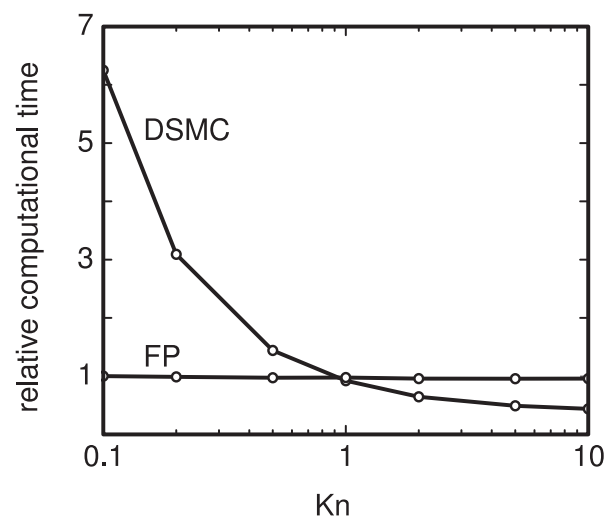

FIG. 14. Relative computational time of DSMC and FP calculations for varying Knudsen number. 
calculations with varying Knudsen number. The Knudsen number is calculated with respect to the reservoir size, while the computing times are normalized to time, required for an FP simulation with $K n=0.01$.

The effort for the DSMC calculations increases sharply with a decreasing Knudsen number, since more and more particle collisions have to be treated. In contrast, the effort for the FP model is nearly not affected by the Knudsen number. For Knudsen numbers of $K n<1$, the FP method becomes clearly more efficient than DSMC.

\section{CONCLUSION}

The kinetic Fokker-Planck ansatz is applied to model a hard sphere gas mixture with an arbitrary number of constituents. The model is constructed to reproduce Grad's 13 moment equations on a Navier-Stokes level of accuracy. To model the associated random process, a stochastic simulation algorithm is derived, which leads to a correct prediction of the species diffusion velocities and temperatures for a homogeneous gas, independent of the applied time step size. Various test cases are studied to show the accuracy of the proposed model, focusing not only on flow quantities of the entire mixture but also on flow quantities of the different species. Within the bounds of expectations, a good agreement between Fokker-Planck simulations and reference DSMC simulations can be observed.

\section{APPENDIX A: BOLTZMANN PRODUCTION TERMS}

Gupta $^{28}$ calculated Boltzmann production terms for the HS collision model assuming a distribution function according to Grad's 13 and 26 moment method. In contrast to expression (23), Gupta evaluated production terms based on the thermal velocities $\hat{\mathbf{c}}^{(\alpha)}$ as defined in (6). Therefore, in the following, all quantities $Q$ marked as $\hat{Q}$ are calculated on the basis of $\hat{\mathbf{c}}^{(\alpha)}$ instead of $\mathbf{c}^{(\alpha)}$. In particular, lower order production terms are given by

$$
\begin{gathered}
P_{\text {Boltz }}^{(\alpha)}\left(\hat{c}_{i}^{(\alpha)}\right)=-\sum_{\beta=1}^{N} v^{(\alpha \beta)} \mu^{(\beta)}\left[\frac{5}{3} \rho^{(\alpha)}\left(u_{i}^{(\alpha)}-u_{i}^{(\beta)}\right)\right. \\
\left.+\frac{1}{6 \hat{\theta}^{(\alpha \beta)}}\left(\hat{h}_{i}^{(\alpha)}-\frac{\rho^{(\alpha)}}{\rho^{(\beta)}} \hat{h}_{i}^{(\beta)}\right)\right], \\
P_{\text {Boltz }}^{(\alpha)}\left(\hat{\mathbf{c}}^{(\alpha)} \hat{\mathbf{c}}^{(\alpha)}\right)=-\sum_{\beta=1}^{N} v^{(\alpha \beta)} \mu^{(\beta)}\left[10 \rho^{(\alpha)} \hat{\theta}^{(\alpha \beta)} \Delta \hat{\theta}^{(\alpha \beta)}\right. \\
\left.-\frac{10}{3}\left(\mu^{(\alpha)}-\mu^{(\beta)}\right) \rho^{(\alpha)} u_{d, i}^{(\alpha)} u_{d, i}^{(\beta)}\right], \\
P_{\text {Boltz }}^{(\alpha)}\left(\hat{c}_{<i}^{(\alpha)} \hat{c}_{j>}^{(\alpha)}\right)=\sum_{\beta=1}^{N} v_{\alpha \beta} \mu^{(\beta)}\left[4 \mu^{(\alpha \beta)}\left\{\hat{\sigma}_{i j}^{(\alpha)}+\frac{1}{3}\left(\hat{\sigma}_{i j}^{(\alpha)}-\frac{\rho^{(\alpha)}}{\rho^{(\beta)}} \hat{\sigma}_{i j}^{(\beta)}\right)\right\}\right. \\
\left.+\frac{10}{3}\left(\mu^{(\alpha)}-\mu^{(\beta)}\right) \hat{\sigma}_{i j}^{(\alpha)}\right],
\end{gathered}
$$

$$
\begin{aligned}
P_{\text {Boltz }}^{(\alpha)}\left(\hat{c}_{i}^{(\alpha)} \hat{\mathbf{c}}^{(\alpha)} \hat{\mathbf{c}}^{(\alpha)}\right)= & -\sum_{\beta=1}^{N} v^{(\alpha \beta)} \mu^{(\beta)}\left[\frac{16}{3} \mu^{(\beta)} \hat{h}_{i}^{(\alpha)}+10\left(\mu^{(\alpha)}-\mu^{(\beta)}\right) \hat{h}_{i}^{\alpha}\right. \\
& +\frac{2}{6} \mu^{(\beta)}\left(5+27 \mu^{(\beta)}\right)\left(\hat{h}_{i}^{(\alpha)}-\frac{\rho^{(\alpha)}}{\rho^{(\beta)}} \hat{h}_{i}^{(\beta)}\right) \\
& \left.+\frac{10}{3} \mu^{(\beta)}\left(5+\mu^{(\beta)}\right) \rho^{(\alpha)} \hat{\theta}^{(\alpha \beta)}\left(u_{i}^{(\alpha)}-u_{i}^{(\beta)}\right)\right] .
\end{aligned}
$$

The values $\mu^{(\alpha)}, \hat{\theta}^{(\alpha)}, \hat{\theta}^{(\alpha \beta)}, \Delta \hat{\theta}^{(\alpha \beta)}$, and $v^{(\alpha \beta)}$ are defined as

$$
\begin{gathered}
\mu^{(\alpha)} \equiv \frac{m^{(\alpha)}}{m^{(\alpha)}+m^{(\beta)}}, \\
\hat{\theta}^{(\alpha)} \equiv \frac{k_{\mathrm{B}} \hat{T}^{(\alpha)}}{m^{(\alpha)}}, \\
\hat{\theta}^{(\alpha \beta)} \equiv \frac{\hat{\theta}^{(\alpha)}+\hat{\theta}^{(\beta)}}{2}, \\
\Delta \hat{\theta}^{(\alpha \beta)} \equiv \frac{\mu^{(\alpha)} \hat{\theta}^{(\alpha)}-\mu^{(\beta)} \hat{\theta}^{(\beta)}}{\hat{\theta}^{(\alpha \beta)}}, \\
\hat{\mathbf{h}}^{(\alpha)} \equiv \hat{\mathbf{q}}^{(\alpha)}-\frac{5}{2} \rho^{(\alpha)} \hat{\theta}^{(\alpha)} \mathbf{u}_{d}^{(\alpha)}, \\
v^{(\alpha \beta)} \equiv \frac{16}{5} \sqrt{\pi} n^{(\beta)}\left(\frac{d^{(\alpha)}+d^{(\beta)}}{2}\right)^{2} \sqrt{\hat{\theta}^{(\alpha \beta)}},
\end{gathered}
$$

where $d^{(\alpha)}$ denotes the diameter of a species $\alpha$ particle. It is worth noting that the right sides of expressions (A1) and (A2) do not vanish, since energy and momentum can be transferred between species. The production terms (A1)-(A4) contain only terms of first order with exception of expression (A2), which additionally contains a second order expression. Since for low Knudsen number flows, only terms of first order are relevant, higher order terms are not required to capture a correct continuum limit. However, the additional term in (A2) is necessary to derive a FP model with a positive diffusion coefficient, as described in Sec. III A. Using the identity

$$
\hat{\mathbf{c}}^{(\alpha)}=\mathbf{c}^{(\alpha)}+\mathbf{u}_{d}^{(\alpha)},
$$

the production terms

$$
\begin{aligned}
& P_{\text {Boltz }}^{(\alpha)}\left(c_{i}^{(\alpha)}\right)=P_{\mathrm{Boltz}}^{(\alpha)}\left(\hat{c}_{i}^{(\alpha)}\right), \\
& P_{\mathrm{Boltz}}^{(\alpha)}\left(\mathbf{c}^{(\alpha)} \mathbf{c}^{(\alpha)}\right)= P_{\mathrm{Boltz}}^{(\alpha)}\left(\hat{\mathbf{c}}^{(\alpha)} \hat{\mathbf{c}}^{(\alpha)}\right)-2 u_{d_{i}}^{(\alpha)} P^{(\alpha)}\left(\hat{c}_{i}^{(\alpha)}\right), \\
& P_{\mathrm{Boltz}}^{(\alpha)}\left(c_{<i}^{(\alpha)} c_{j>}^{(\alpha)}\right)= P_{\mathrm{Boltz}}^{(\alpha)}\left(\hat{c}_{<i}^{(\alpha)} \hat{c}_{j>}^{(\alpha)}\right)-u_{d<j}^{(\alpha)} P_{\mathrm{Boltz}}^{(\alpha)}\left(\hat{c}_{i>}^{(\alpha)}\right) \\
&-u_{d<i}^{(\alpha)} P_{\mathrm{Boltz}}^{(\alpha)}\left(\hat{c}_{j>}^{(\alpha)}\right), \\
& P_{\mathrm{Boltz}}^{(\alpha)}\left(c_{i}^{(\alpha)} \mathbf{c}^{(\alpha)} \mathbf{c}^{(\alpha)}\right)= P_{\mathrm{Boltz}}^{(\alpha)}\left(\hat{c}_{i}^{(\alpha)} \hat{\mathbf{c}}^{(\alpha)} \hat{\mathbf{c}}^{(\alpha)}\right)-2 u_{d_{j}}^{(\alpha)} P_{\mathrm{Boltz}}^{(\alpha)}\left(\hat{c}_{i}^{(\alpha)} \hat{c}_{j}^{(\alpha)}\right) \\
&+u_{d_{j}}^{(\alpha)} u_{d_{j}}^{(\alpha)} P_{\mathrm{Boltz}}^{(\alpha)}\left(\hat{c}_{i}^{(\alpha)}\right) \\
&-u_{d_{i}}^{(\alpha)} P_{\mathrm{Boltz}}^{(\alpha)}\left(\hat{c}_{i}^{(\alpha)} \hat{\mathbf{c}}^{(\alpha)} \hat{\mathbf{c}}^{(\alpha)}\right) \\
&+2 u_{d_{i}}^{(\alpha)} u_{d_{j}}^{(\alpha)} P_{\mathrm{Boltz}}^{(\alpha)}\left(\hat{c}_{j}^{(\alpha)}\right)
\end{aligned}
$$


can be transformed into a $\mathbf{c}$ basis. When we neglected the second term in expression (A1), expression (A13) approximately becomes

$$
\begin{aligned}
P^{(\alpha)}\left(\mathbf{c}^{(\alpha)} \mathbf{c}^{(\alpha)}\right)= & -10 \sum_{\beta=1}^{N} v^{(\alpha \beta)} \mu^{(\beta)} \rho^{(\alpha)}\left[\frac{k_{\mathrm{B}}}{m^{(\alpha)}+m^{(\beta)}}\left(T^{(\alpha)}-T^{(\beta)}\right)\right. \\
& \left.-\frac{\mu^{(\beta)}}{3}\left(\mathbf{u}^{(\alpha)}-\mathbf{u}^{(\beta)}\right)^{2}\right] .
\end{aligned}
$$

Expression (A16) is employed in Sec. III A for the construction of the linear FP operator. Therefore, the linear FP operator may not reproduce the production terms (A13) when the heat fluxes $\hat{h}_{i}^{(\alpha)}$ become large and the second term in expression (A1) becomes dominant. However, the higher order model, constructed in Sec. III B, reproduces the full production term (A13).

\section{APPENDIX B: FOKKER-PLANCK PRODUCTION TERMS}

For the FP-operator, production terms (13) can be calculated independently of a distribution function. For the following derivations, it is only assumed that $D^{(\alpha)}$ does not depend on microscopic particle velocities $\mathbf{v}^{(\alpha)}$ and that the distribution function tends to zero for large velocities sufficiently fast. Hence, the identities

$$
\begin{gathered}
\lim _{v_{i}^{(\alpha)} \rightarrow 0} f^{(\alpha)}=0, \\
\lim _{v_{i}^{(\alpha)} \rightarrow 0} \frac{\partial f^{(\alpha)}}{\partial v_{i}^{(\alpha)}}=0
\end{gathered}
$$

must hold. The meaning of sufficiently fast depends on the production term and will be discussed later in this section. Applying definition (13), it can be calculated

$$
\begin{aligned}
P_{\mathrm{FP}}^{(\alpha)}(X)= & -m^{(\alpha)} \int \underbrace{\frac{\partial}{\partial v_{j}^{(\alpha)}}\left(f^{(\alpha)} A_{j}^{(\alpha)}\right) X}_{I} \mathrm{~d} \mathbf{c}^{(\alpha)} \\
& +m^{(\alpha)} D^{(\alpha)} \int \underbrace{\frac{\partial^{2} f^{(\alpha)}}{\partial v_{j}^{(\alpha)} \partial v_{j}^{(\alpha)}} X}_{I I} \mathrm{~d} \mathbf{c}^{(\alpha)} .
\end{aligned}
$$

Term $I$ can be written as

$$
I=\frac{\partial}{\partial v_{j}^{(\alpha)}}\left(f^{(\alpha)} A_{j}^{(\alpha)} X\right)-\frac{\partial X}{\partial v_{j}^{(\alpha)}} A_{j}^{(\alpha)} f^{(\alpha)} .
$$

Similarly, term II can be written as

$$
\begin{gathered}
I I=\frac{\partial}{\partial v_{j}^{(\alpha)}}\left(\frac{\partial f^{(\alpha)}}{\partial v_{j}^{(\alpha)}} X\right)-\frac{\partial X}{\partial v_{j}^{(\alpha)}} \frac{\partial f^{(\alpha)}}{\partial v_{j}^{(\alpha)}} \\
=\frac{\partial}{\partial v_{j}^{(\alpha)}}\left(\frac{\partial f^{(\alpha)}}{\partial v_{j}^{(\alpha)}} X\right)-\frac{\partial}{\partial v_{j}^{(\alpha)}}\left(f^{(\alpha)} \frac{\partial X}{\partial v_{j}^{(\alpha)}}\right)+f^{(\alpha)} \frac{\partial^{2} X}{\partial v_{j}^{(\alpha)} \partial v_{j}^{(\alpha)}} .
\end{gathered}
$$

Hence, for the production terms, follow

$$
\begin{aligned}
P_{\mathrm{FP}}^{(\alpha)}(X)= & -\left.m^{(\alpha)} f^{(\alpha)} A_{j}^{(\alpha)} X\right|_{-\infty} ^{\infty}+m^{(\alpha)} \int \frac{\partial X}{\partial v_{j}^{(\alpha)}} A_{j}^{(\alpha)} f^{(\alpha)} \mathrm{d} \mathbf{c}^{(\alpha)} \\
& +\left.m^{(\alpha)} D^{(\alpha)} \frac{\partial f^{(\alpha)}}{\partial v_{j}^{(\alpha)}} X\right|_{-\infty} ^{\infty}-\left.m^{(\alpha)} D^{(\alpha)} f^{(\alpha)} \frac{\partial X}{\partial v_{j}^{(\alpha)}}\right|_{-\infty} ^{\infty} \\
& +m^{(\alpha)} D^{(\alpha)} \int f^{(\alpha)} \frac{\partial^{2} X}{\partial v_{j}^{(\alpha)} \partial v_{j}^{(\alpha)}} \mathrm{d} \mathbf{c}^{(\alpha)}
\end{aligned}
$$

Assuming that the distribution function and its derivative vanish sufficiently fast for large velocities, the first, third, and fourth terms in (B7) can be neglected.

Sufficiently fast means that the distribution function vanishes faster for large velocities than the terms $A_{j}^{(\alpha)} X, X$, and $\partial X / \partial v_{j}^{(\alpha)}$ might increase. This assumption is generally difficult to prove. However, in this work, $A_{j}^{(\alpha)} X, X$, and $\partial X / \partial v_{j}^{(\alpha)}$ feature a simple polynomial form. In addition, only production terms for low Knudsen number flows are relevant. Hence, the distribution function

$$
f^{(\alpha)} \sim \exp \left(-c_{i}^{(\alpha)} c_{i}^{(\alpha)}\right)
$$

can be expanded around a Maxwell distribution ${ }^{28}$ and features an exponential dependency of microscopic velocities. As a result, the first, third, and fourth term in (B7) vanish. For the production terms, follow

$$
P_{\mathrm{FP}}^{(\alpha)}(X)=m^{(\alpha)}\left\langle\frac{\partial X}{\partial v_{j}^{(\alpha)}} A_{j}^{(\alpha)} \mid f^{(\alpha)}\right\rangle+m^{(\alpha)} D^{(\alpha)}\left\langle\frac{\partial^{2} X}{\partial v_{j}^{(\alpha)} \partial v_{j}^{(\alpha)}} \mid f^{(\alpha)}\right\rangle .
$$

In particular, it can be found

$$
\begin{gathered}
P_{\mathrm{FP}}^{(\alpha)}\left(c_{i}^{(\alpha)}\right)=m^{(\alpha)}\left\langle A_{i}^{(\alpha)} \mid f^{(\alpha)}\right\rangle \\
P_{\mathrm{FP}}^{(\alpha)}\left(\mathbf{c}^{(\alpha)} \mathbf{c}^{(\alpha)}\right)=2 m^{(\alpha)}\left\langle A_{i}^{(\alpha)} c_{i}^{(\alpha)} \mid f^{(\alpha)}\right\rangle+6 \rho^{(\alpha)} D^{(\alpha)}, \\
P_{\mathrm{FP}}^{(\alpha)}\left(c_{i}^{(\alpha)} c_{j}^{(\alpha)}\right)=m^{(\alpha)}\left\langle A_{i}^{(\alpha)} c_{j}^{(\alpha)} \mid f^{(\alpha)}\right\rangle+m^{(\alpha)}\left\langle A_{j}^{(\alpha)} c_{i}^{(\alpha)} \mid f^{(\alpha)}\right\rangle \\
+2 \delta_{i j} \rho^{(\alpha)} D^{(\alpha)}, \\
P_{\mathrm{FP}}^{(\alpha)}\left(c_{i}^{(\alpha)} \mathbf{c}^{(\alpha)} \mathbf{c}^{(\alpha)}\right)=m^{(\alpha)}\left\langle A_{i}^{(\alpha)} c_{j}^{(\alpha)} c_{j}^{(\alpha)} \mid f^{(\alpha)}\right\rangle \\
+2 m^{(\alpha)}\left\langle A_{j}^{(\alpha)} c_{j}^{(\alpha)} c_{i}^{(\alpha)} \mid f^{(\alpha)}\right\rangle .
\end{gathered}
$$

\section{APPENDIX C: LINEAR SYSTEM OF EQUATIONS}

The nine parameters $\gamma_{i}^{(\alpha)}$ and $\psi_{i j}^{(\alpha)}$ occurring in the higherorder model (31) are calculated as a solution of a linear system of equations. 
Combination of (33) with (B13) results in three linear equations

$$
\begin{aligned}
K_{i}^{(\alpha)} & \left\langle c_{j}^{(\alpha)} c_{j}^{(\alpha)} \mid f^{(\alpha)}\right\rangle+2 K_{j}^{(\alpha)}\left\langle c_{i}^{(\alpha)} c_{j}^{(\alpha)} \mid f^{(\alpha)}\right\rangle-3 s^{(\alpha)}\left\langle c_{i}^{(\alpha)} c_{j}^{(\alpha)} c_{j}^{(\alpha)} \mid f^{(\alpha)}\right\rangle \\
& +\psi_{i l}^{(\alpha)}\left\langle c_{l}^{(\alpha)} c_{j}^{(\alpha)} c_{j}^{(\alpha)} \mid f^{(\alpha)}\right\rangle+2 \psi_{j l}^{(\alpha)}\left\langle c_{j}^{(\alpha)} c_{l}^{(\alpha)} c_{i}^{(\alpha)} \mid f^{(\alpha)}\right\rangle \\
& +\gamma_{i}^{(\alpha)}\left[\left\langle c_{l}^{(\alpha)} c_{l}^{(\alpha)} c_{j}^{(\alpha)} c_{j}^{(\alpha)} \mid f^{(\alpha)}\right\rangle-\left\langle c_{l}^{(\alpha)} c_{l}^{(\alpha)} \mid f^{(\alpha)}\right\rangle\left\langle c_{j}^{(\alpha)} c_{j}^{(\alpha)} \mid f^{(\alpha)}\right\rangle\right] \\
& +2 \gamma_{j}^{(\alpha)}\left[\left\langle c_{l}^{(\alpha)} c_{l}^{(\alpha)} c_{j}^{(\alpha)} c_{i}^{(\alpha)} \mid f^{(\alpha)}\right\rangle-\left\langle c_{l}^{(\alpha)} c_{l}^{(\alpha)} \mid f^{(\alpha)}\right\rangle\right. \\
& \left.\times\left\langle c_{j}^{(\alpha)} c_{i}^{(\alpha)} \mid f^{(\alpha)}\right\rangle\right]=\frac{1}{m^{(\alpha)}} P_{\mathrm{Boltz}}^{(\alpha)}\left(c_{i}^{(\alpha)} c^{(\alpha)} c^{(\alpha)}\right) .
\end{aligned}
$$

Combining Eq. (32) with expression (B12) yields six linear equations

$$
\begin{aligned}
-2 s^{(\alpha)} & \left\langle c_{i}^{(\alpha)} c_{j}^{(\alpha)} \mid f^{(\alpha)}\right\rangle+\psi_{i l}^{(\alpha)}\left\langle c_{j}^{(\alpha)} c_{l}^{(\alpha)} \mid f^{(\alpha)}\right\rangle+\psi_{j l}^{(\alpha)}\left\langle c_{i}^{(\alpha)} c_{l}^{(\alpha)} \mid f^{(\alpha)}\right\rangle \\
& +\gamma_{i}^{(\alpha)}\left\langle c_{j}^{(\alpha)} c_{l}^{(\alpha)} c_{l}^{(\alpha)} \mid f^{(\alpha)}\right\rangle+\gamma_{j}^{(\alpha)}\left\langle c_{i}^{(\alpha)} c_{l}^{(\alpha)} c_{l}^{(\alpha)} \mid f^{(\alpha)}\right\rangle+2 \delta_{i j} D^{(\alpha)} \\
= & \frac{1}{m^{(\alpha)}}\left(P_{\text {Boltz }}^{(\alpha)}\left(c_{<i}^{(\alpha)} c_{j>}^{(\alpha)}\right)+\frac{1}{3} \delta_{i j} P_{\text {Boltz }}^{(\alpha)}\left(c^{(\alpha)} c^{(\alpha)}\right)\right) .
\end{aligned}
$$

In a kinetic FP simulation, the nine linear equations (C2) and (C1) are solved at each time step and each grid cell to calculate the model parameters $\psi_{i j}^{(\alpha)}$ and $\gamma_{i}^{\alpha}$.

\section{APPENDIX D: TIME EVOLUTION OF MOMENTS}

To integrate the stochastic equation of motion (34), the species velocities and temperatures at the end of the integration process must be known. When assuming homogeneous systems, the rate of change of velocity moments is given by production terms. ${ }^{43}$ Hence, the system

$$
\frac{\mathrm{d}}{\mathrm{d} t}\left(\begin{array}{c}
v_{0}^{(\alpha)} \\
v_{1}^{(\alpha)} \\
v_{2}^{(\alpha)} \\
T^{(\alpha)} \\
q_{0}^{(\alpha)} \\
q_{1}^{(\alpha)} \\
q_{1}^{(\alpha)}
\end{array}\right)=\left(\begin{array}{c}
P_{\mathrm{Boltz}}^{(\alpha)}\left(c_{0}^{(\alpha)}\right) \\
P_{\mathrm{Boltz}}^{(\alpha)}\left(c_{1}^{(\alpha)}\right) \\
P_{\mathrm{Boltz}}^{(\alpha)}\left(c_{2}^{(\alpha)}\right) \\
\frac{1}{3 n^{(\alpha)} k_{\mathrm{B}}} P_{\mathrm{Boltz}}^{(\alpha)}\left(\mathbf{c}^{(\alpha)} \mathbf{c}^{(\alpha)}\right) \\
\frac{1}{2} P_{\mathrm{Boltz}}^{(\alpha)}\left(c_{0}^{(\alpha)} \mathbf{c}^{(\alpha)} \mathbf{c}^{(\alpha)}\right) \\
\frac{1}{2} P_{\mathrm{Boltz}}^{(\alpha)}\left(c_{1}^{(\alpha)} \mathbf{c}^{(\alpha)} \mathbf{c}^{(\alpha)}\right) \\
\frac{1}{2} P_{\mathrm{Boltz}}^{(\alpha)}\left(c_{2}^{(\alpha)} \mathbf{c}^{(\alpha)} \mathbf{c}^{(\alpha)}\right)
\end{array}\right)
$$

of ordinary differential equations can be deduced for each species. Note that the right side of (D1) depends on all species in the mixture. Hence, expression (D1) designates a system of $7 \times N_{\text {species }}$ differential equations, where $N_{\text {species }}$ denotes the number of species. The evolution equations for the heat fluxes are included in system (D1), since the production terms (A1) also depend on heat fluxes. When the vector $\left(\mathbf{u}^{(\alpha), n}, T^{(\alpha), n}, \mathbf{q}^{(\alpha), n}\right)$ is employed as an initial condition, the solution of (D1) yields the species flow velocities and temperatures $\left(\mathbf{u}^{(\alpha), n+1}, T^{(\alpha), n+1}, \mathbf{q}^{(\alpha), n+1}\right)$ at the end of the time step.

\section{REFERENCES}

1'I. D. Boyd, "Computation of neutral gas flow from a hall thruster into a vacuum chamber,” AIP Conf. Proc. 663, 541 (2003).

${ }^{2}$ M. S. Ivanov, G. N. Markelov, S. F. Gimelshein, L. V. Mishina, A. N. Krylov, and N. V. Grechko, "High-altitude capsule aerodynamics with real gas effects," J. Spacecr. Rockets 35, 16-22 (1998).
${ }^{3}$ T. Huismann and I. Boyd, "Simulation of hall thruster plumes in a vacuum chamber using a hybrid method," in 44th AIAA/ASME/SAE/ASEE Joint Propulsion Conference Exhibit, 2008.

${ }^{4} \mathrm{~J}$. Anderson, Hypersonic and High-Temperature Gas Dynamics, AIAA Education, 2nd ed. (AIAA, 2006).

${ }^{\mathbf{5}}$ J. O. Hirschfelder, C. F. Curtiss, and R. Bird, Molecular Theory of Gases and Liquids (Wiley, New York, 1954).

${ }^{6}$ G. A. Bird, Molecular Gas Dynamics and the Direct Simulation of Gas Flows, Oxford Engineering Science Series (Clarendon Press, 1994).

${ }^{7}$ W. Wagner, “A convergence proof for bird's direct simulation Monte Carlo method for the Boltzmann equation," J. Stat. Phys. 66, 1011-1044 (1992).

${ }^{8}$ I. D. Boyd, "Analysis of rotational nonequilibrium in standing shock waves of nitrogen,” AIAA J. 28, 1997-1999 (1990).

${ }^{9}$ I. D. Boyd, “Analysis of vibrational-translational energy transfer using the direct simulation Monte Carlo method,” Phys. Fluids A 3, 1785-1791 (1991).

${ }^{10}$ K. Breuer, E. Piekos, and D. Gonzales, "DSMC simulations of continuum flows," in 30th Thermophysics Conference (American Institute of Aeronautics and Astronautics, 1995).

${ }^{11}$ D. Wadsworth and D. Erwin, "One-dimensional hybrid continuum/particle simulation approach for rarefied hypersonic flows," in 5th Joint Thermophysics and Heat Transfer Conference (American Institute of Aeronautics and Astronautics, 1990).

${ }^{12}$ T. E. Schwartzentruber, L. C. Scalabrin, and I. D. Boyd, "A modular particlecontinuum numerical method for hypersonic non-equilibrium gas flows," J. Comput. Phys. 225, 1159-1174 (2007).

${ }^{13}$ P. Jenny, M. Torrilhon, and S. Heinz, "A solution algorithm for the fluid dynamic equations based on a stochastic model for molecular motion," J. Comput. Phys. 229, 1077-1098 (2010).

${ }^{14} \mathrm{~J}$. Burt and I. Boyd, "A low diffusion particle method for simulating compressible inviscid flows," in 39th AIAA Thermophysics Conference (American Institute of Aeronautics and Astronautics, 2007).

${ }^{15}$ M. N. Macrossan, “A particle-only hybrid method for near-continuum flows," AIP Conf. Proc. 585, 388 (2001).

${ }^{16}$ M. Pfeiffer, A. Mirza, and P. Nizenkov, "Evaluation of particle-based continuum methods for a coupling with the direct simulation Monte Carlo method based on a nozzle expansion," Phys. Fluids 31, 073601 (2019).

${ }^{17}$ M. H. Gorji, M. Torrilhon, and P. Jenny, "Fokker-Planck model for computational studies of monatomic rarefied gas flows," J. Fluid Mech. 680, 574-601 (2011).

${ }^{18}$ M. H. Gorji and M. Torillhon, "A Fokker-Planck model of hard sphere gases based on H-theorem,” AIP Conf. Proc. 1786, 090001 (2016).

${ }^{19}$ J. Mathiaud and L. Mieussens, "A Fokker-Planck model of the Boltzmann equation with correct Prandtl number," J. Stat. Phys. 162, 397-414 (2015).

${ }^{20}$ S. K. Singh and S. Ansumali, "Fokker-planck model of hydrodynamics," Phys. Rev. E 91, 033303 (2015).

${ }^{21}$ H. Gorji and P. Jenny, "A kinetic model for gas mixtures based on a FokkerPlanck equation,” J. Phys.: Conf. Ser. 362, 012042 (2012).

${ }^{22}$ M. H. Gorji and P. Jenny, "A Fokker-Planck based kinetic model for diatomic rarefied gas flows," Phys. Fluids 25, 062002 (2013).

${ }^{23}$ M. Sadr and M. H. Gorji, "A continuous stochastic model for non-equilibrium dense gases," Phys. Fluids 29, 122007 (2017).

${ }^{24}$ M. H. Gorji and P. Jenny, "An efficient particle Fokker-Planck algorithm for rarefied gas flows," J. Comput. Phys. 262, 325-343 (2014).

${ }^{25} \mathrm{M}$. H. Gorji and P. Jenny, "Fokker-Planck-DSMC algorithm for simulations of rarefied gas flows,” J. Comput. Phys. 287, 110-129 (2015).

${ }^{26}$ J. M. Burgers, Flow equations for composite gases (Academic Press, New York, 1969).

${ }^{27}$ G. M. Kremer, An Introduction to the Boltzmann Equation and Transport Processes in Gases (Springer Berlin Heidelberg, 2010).

${ }^{28}$ V. K. Gupta, "Mathematical modeling of rarefied gas-mixtures," Ph.D. thesis, RWTH Aachen, 2015.

${ }^{29}$ G. A. Bird, "Monte-Carlo simulation in an engineering context," Prog. Astronaut. Aeronaut. 74, 239-255 (1981). 
${ }^{30} \mathrm{~K}$. Koura and H. Matsumoto, "Variable soft sphere molecular model for inverse-power-law or Lennard-Jones potential," Phys. Fluids A 3, 2459-2465 (1991).

${ }^{31} \mathrm{~K}$. Koura and H. Matsumoto, "Variable soft sphere molecular model for air species," Phys. Fluids A 4, 1083-1085 (1992).

${ }^{32}$ H. A. Hassan and D. B. Hash, "A generalized hard-sphere model for Monte Carlo simulation," Phys. Fluids A 5, 738-744 (1993).

${ }^{33}$ E. Goldman, “Equations for gas mixtures," Phys. Fluids 10, 1928 (1967).

${ }^{34}$ S. Chapman and T. G. owling, The Mathematical Theory of Non-uniform Gases (The Syndics of the Cambridge University Press, 1960).

${ }^{35}$ C. W. Gardiner, Handbook of Stochastic Methods for Physics, Chemistry, and the Natural Sciences, Springer Series in Synergetics (Springer-Verlag, 1983).

${ }^{36}$ F. Fei, Z. Liu, J. Zhang, and C. Zheng, "A particle Fokker-Planck algorithm with multiscale temporal discretization for rarefied and continuum gas flows," Commun. Comput. Phys. 22, 338-374 (2017).

${ }^{37}$ P. Jenny, M. Muradoglu, K. Liu, S. B. Pope, and D. A. Caughey, "Pdf simulations of a bluff-body stabilized flow,” J. Comput. Phys. 169, 1-23 (2001).
${ }^{38} \mathrm{P}$. Jenny, S. Küchlin, and H. Gorji, "Controlling the bias error of Fokker-Planck methods for rarefied gas dynamics simulations," Phys. Fluids 31, 062005 (2019).

${ }^{39}$ M. A. Gallis, J. R. Torczynski, S. J. Plimpton, D. J. Rader, and T. Koehler, "Direct simulation Monte Carlo: The quest for speed," AIP Conf. Proc. 1628, 27-36 (2014).

${ }^{40}$ S. J. Plimpton, S. G. Moore, A. Borner, A. K. Stagg, T. P. Koehler, J. R. Torczynski, and M. A. Gallis, "Direct simulation Monte Carlo on petaflop supercomputers and beyond," Phys. Fluids 31, 086101 (2019).

${ }^{41}$ M. A. Gallis, T. P. Koehler, J. R. Torczynski, and S. J. Plimpton, "Direct simulation Monte Carlo investigation of the Richtmyer-Meshkov instability," Phys. Fluids 27, 084105 (2015).

${ }^{42}$ R. Prakash, S. Gai, and S. O’Byrne, "A direct simulation Monte Carlo study of hypersonic leading-edge separation with rarefaction effects," Phys. Fluids 30, 063602 (2018).

${ }^{43}$ V. K. Gupta and M. Torrilhon, "Reprint of: Comparison of relaxation phenomena in binary gas-mixtures of Maxwell molecules and hard spheres," Comput. Math. Appl. 72, 271-287 (2016). 